\title{
Nijenhuis Operators on 3-Hom-L-dendriform algebras
}

\author{
A. Ben Hassine $e^{1,2}$; T. Chtioui ${ }^{2}+$ S. Mabrouk ${ }^{3} \ddagger$ \\ ${ }^{1}$ Department of Mathematics, Faculty of Science and Arts at Belqarn, P. O. Box 60, Sabt Al-Alaya 61985, \\ University of Bisha, Kingdom of Saudi Arabia \\ 2 Faculty of Sciences, University of Sfax, BP 1171, 3000 Sfax, Tunisia \\ ${ }^{3}$ Faculty of Sciences, University of Gafsa, BP 2100, Gafsa, Tunisia
}

\begin{abstract}
The goal of this work is to introduce the notion of 3-Hom-Lie-dendriform algebras which is the dendriform version of 3-Hom-Lie-algebras. They can be also regarded as the ternary analogous of Hom-Lie-dendriform algebras. We give the representation of a 3-Hom-pre-Lie algebra. Moreover, we introduce the notion of Nijenhuis operators on a 3-Hom-pre-Lie algebra and provide some constructions of 3-Hom-Lie-dendriform algebras in term of Nijenhuis operators. Parallelly, we introduce the notion of a product and complex structures on a 3-HomLie-dendriform algebras and there are also four types special integrability conditions.
\end{abstract}

Keywords: 3-Hom-L-dendriform algebras, $O$-operators, symplectic structure, Nijenhuis operator, complex structure, product structure.

MSC(2010): 17A40; 17B70.

\section{Contents}

1 Preliminaries and basics

2 Representations of 3-Hom-pre-Lie algebras

3 3-Hom-L-dendriform algebras 9

4 Nijenhuis Operators on 3-Hom-L-dendriform algebras 15

4.1 Second-order deformation of 3-Hom-L-dendriform algebras . . . . . . . . . . . . 15

4.2 Some properties of Nijenhuis operators . . . . . . . . . . . . . . . . 17

4.3 Product and complex structures on 3-Hom-L-dendriform algebras . . . . . . . . . . 17

\section{Introduction}

Hom-Lie algebras were introduced in the context of q-deformation of Witt and Virasoro algebras. In a sequel, various concepts and properties have been derived to the framework of other homalgebras as well. The study of Hom-algebras appeared extensively in the work of J. Hartwig, D.

\footnotetext{
${ }^{*}$ Corresponding author, E-mail: benhassine.abdelkader@yahoo.fr

${ }^{+}$Corresponding author, E-mail: chtioui.taoufik@yahoo.fr

‡Corresponding author, E-mail: mabrouksami00@yahoo.fr
} 
Larsson, A. Makhlouf, S. Silvestrov, D. Yau and other authors ([3, 21, 31, 32, 41]). Other types of algebras (such as associative, Leibniz, Poisson, Hopf,. . .) twisted by homomorphisms have also been studied in the last few years.

Recently, there have been several interesting developments of Hom-Lie algebras in mathematics and mathematical physics, including Hom-Lie bialgebras [12, 13], quadratic Hom-Lie algebras [10], involutive Hom-semigroups [44], deformed vector fields and differential calculus [28], representations [33, 43], cohomology and homology theory [3, 41], Yetter-Drinfeld categories [39], Hom-Yang-Baxter equations [14, 15, 29, 33, 42], Hom-Lie 2-algebras [36, 37], $(m, n)$-Hom-Lie algebras [30], Hom-left-symmetric algebras [31] and enveloping algebras [26].

The twisted version of another type algebras, called dendriform algebras. These algebras were introduced by Loday as Koszul dual of associative dialgebras [24]. Free dendriform algebra over a vector space has been constructed by using planar binary trees. Dendriform algebras also arise from Rota-Baxter operators on some associative algebra [1]. Recently, an explicit cohomology theory for dendriform algebras has been introduced and the formal deformation theory for dendriform algebras (as well as coalgebras) has been studied in [17, 18]. The cohomology involves certain combinatorial maps. Some results in 3-L-dendriform algebras are given in [11].

The twisted version of dendriform structures, called Hom-dendriform structures was introduced in [22]. These algebras can be thought of as splitting of Hom-associative algebras. They also arise from Rota-Baxter operator on Hom-associative algebras. In [30] the authors study Homdendriform algebras from the point of view of monoidal categories. In [16] is given the twist of the construction of [17] by a homomorphism to formulate a cohomology for Hom-dendriform algebras.

Deformations of $n$-Lie algebras have been studied from several aspects. See [9, 19] for more details. In particular, a notion of a Nijenhuis operator on a 3-Lie algebra was introduced in [45] in the study of the 1-order deformations of a 3-Lie algebra. But there are some quite strong conditions in this definition of a Nijenhuis operator. In the case of Lie algebras, one could obtain fruitful results by considering one-parameter infinitesimal deformations, i.e. 1-order deformations. However, for $n$-Lie algebras, we believe that one should consider (n - 1)-order deformations to obtain similar results. In [19], for 3- Lie algebras, the author had already considered 2-order deformations. For the case of pre-Lie algebras, the authors in [38] give the notion of Nijenhuis operator.

Thus it is time to study 3-Hom-Lie-dendriform algebras, 3-Hom-pre-Lie algebras and Nijenhuis operator. Similarly, we give the some proprieties of Nijenhuis operator on 3-Hom-Lie-dendriform algebras and the relationship between the Nijenhuis operator on 3-Hom-Lie-dendriform algebras and the Nijenhuis operator on 3-Hom-pre-Lie algebras.

Product structures and complex structures on a 3-Hom-Lie algebra can be viewed as special Nijenhuis operator([34]). These structures were considered by many authors from different points of view. For example, A. Andrada and Aderián studied Complexproduct structures on 6-dimensional nilpotent Lie algebras([6]), A. Andrada, M. L. Barberis andI. Dotti studied Classification of abelian complex structures on 6-dimensional Lie algebras([4]).A. Andrada, M. L. Barberis and I. Dotti studied Product structures on four dimensional solvable Lie algebras([5]).

This paper is organized as follows: In Section 1, we recall the concepts of 3-Hom-Lie algebra and introduce the notion of 3-Hom-pre-Lie algebra, representation of 3-Hom-pre-Lie algebra. Some results and the definition of 3-Hom-L-dendriform are established in Section 2. Section 3 is dedicated to study the second order deformation of 3-Hom-Lie-dendriform and introduce the notion of Nijenhuis operator on 3-Hom-Lie-dendriform, which could generate a trivial deformation. In the other part of this section we give some properties and results of Nijenhuis operators. Finally, we introduce the notion of product and complex structures on a 3-Hom-L-dendriform algebra and 
prove that a it is the direct sum of two Hom-subalgebras (as vector spaces) if and only if there is a product (resp. complex) structure on it. Next, we study several special product and complex structures, which are called strict product (complex) structures, abelian product (complex) structures, strong abelian product (complex) structures and perfect product (complex) structures, respectively.

All vector spaces are considered over field $\mathbb{K}$ of characteristic 0 . Moreover all ternary Homalgebras are supposed multiplicative, that is the twisted map preserved ternary operations.

\section{Preliminaries and basics}

In this section, we give some general results about 3-Hom-Lie algebras (see [8]). First, recall that a 3-Hom-Lie algebra is a vector space $A$ together with a skew-symmetric linear map (3-Hom-Lie bracket) $[\cdot, \cdot, \cdot]: \otimes^{3} A \rightarrow A$ and a linear map $\alpha: A \rightarrow A$ such that the following fundamental identity (FI) holds:

$$
\begin{aligned}
& {\left[\alpha\left(x_{1}\right), \alpha\left(x_{2}\right),\left[x_{3}, x_{4}, x_{5}\right]\right]=\left[\left[x_{1}, x_{2}, x_{3}\right], \alpha\left(x_{4}\right), \alpha\left(x_{5}\right)\right]} \\
& +\left[\alpha\left(x_{3}\right),\left[x_{1}, x_{2}, x_{4}\right], \alpha\left(x_{5}\right)\right]+\left[\alpha\left(x_{3}\right), \alpha\left(x_{4}\right),\left[x_{1}, x_{2}, x_{5}\right]\right]
\end{aligned}
$$

for $x_{i} \in A, 1 \leq i \leq 5$. If $\alpha[\cdot, \cdot, \cdot]=[\cdot, \cdot, \cdot] \circ \alpha^{\otimes^{3}}$, we say that $A$ is multiplicative. Define for any $x_{1}, x_{2} \in A$, the linear map

$$
\text { ad }: A \wedge A \rightarrow g l(A), \quad \operatorname{ad}\left(x_{1}, x_{2}\right)(x):=\left[x_{1}, x_{2}, x\right], \quad \forall x \in A .
$$

The identity can be written as follows:

$$
\begin{aligned}
\operatorname{ad}\left(\alpha\left(x_{1}\right), \alpha\left(x_{2}\right)\right)\left[x_{3}, x_{4}, x_{5}\right] & =\left[a d\left(x_{1}, x_{2}\right)\left(x_{3}\right), \alpha\left(x_{4}\right), \alpha\left(x_{5}\right)\right]+\left[\alpha\left(x_{3}\right),\left(x_{1}, x_{2}\right)\left(x_{4}\right), \alpha\left(x_{5}\right)\right] \\
& +\left[\alpha\left(x_{3}\right), \alpha\left(x_{4}\right), a d\left(x_{1}, x_{2}\right)\left(x_{5}\right)\right]
\end{aligned}
$$

for all $x_{1}, x_{2}, x_{3} \in A$. A morphism of 3 -Hom-Lie algebras $\phi:\left(A_{1},[\cdot, \cdot, \cdot]_{A_{1}}, \alpha_{1}\right) \rightarrow\left(A_{2},[\cdot, \cdot, \cdot]_{A_{2}}, \alpha_{2}\right)$ is a linear map such that for all $x_{1}, x_{2}, x_{3} \in A$,

$$
\begin{aligned}
\phi\left(\left[x_{1}, x_{2}, x_{3}\right]_{A_{1}}\right) & =\left[\phi\left(x_{1}\right), \phi\left(x_{2}\right), \phi\left(x_{3}\right)\right]_{A_{2}}, \\
\phi \circ \alpha_{1} & =\alpha_{2} \circ \phi .
\end{aligned}
$$

The notion of a representation of an $n$-Hom-Lie algebra was introduced in [2].

Definition 1.1. Let $(A,[\cdot, \cdot, \cdot], \alpha)$ be a 3-Hom-Lie algebra, $V$ be a vector space and $\phi: V \rightarrow V$ be a linear map. A representation of $A$ on $V$ with respect to $\phi$ is a linear map $\rho: \wedge^{2} A \rightarrow g l(V)$ such that

$$
\begin{aligned}
& \phi \rho\left(x_{1}, x_{2}\right)=\rho\left(\alpha\left(x_{1}\right), \alpha\left(x_{2}\right)\right) \phi, \\
& \rho\left(\alpha\left(x_{1}\right), \alpha\left(x_{2}\right)\right) \rho\left(x_{3}, x_{4}\right)-\rho\left(\alpha\left(x_{3}\right), \alpha\left(x_{4}\right)\right) \rho\left(x_{1}, x_{2}\right) \\
& =\rho\left(\left[x_{1}, x_{2}, x_{3}\right], \alpha\left(x_{4}\right)\right) \circ \phi-\rho\left(\left[x_{1}, x_{2}, x_{4}\right], \alpha\left(x_{3}\right)\right) \circ \phi, \\
& \rho\left(\left[x_{1}, x_{2}, x_{3}\right], \alpha\left(x_{4}\right)\right) \circ \phi=\rho\left(\alpha\left(x_{1}\right), \alpha\left(x_{2}\right)\right) \rho\left(x_{3}, x_{4}\right) \\
& +\rho\left(\alpha\left(x_{2}\right), \alpha\left(x_{3}\right)\right) \rho\left(x_{1}, x_{4}\right)+\rho\left(\alpha\left(x_{3}\right), \alpha\left(x_{1}\right)\right) \rho\left(x_{2}, x_{4}\right)
\end{aligned}
$$

for $x_{i} \in A, 1 \leq i \leq 4$. We say that $(V, \rho, \phi)$ is a representation of $A$ or $V$ is an $A$-module.

The tuple $(A, a d, \alpha)$ is a representation of $A$ on itself which is called the adjoint representation. 
Lemma 1.1. Let $(A,[\cdot, \cdot, \cdot], \alpha)$ be a $3-H o m$-Lie algebra, $V$ a vector space, $\phi \in \operatorname{End}(V)$ and $\rho: \wedge^{2} A \rightarrow g l(V)$ a linear map. Then $(V, \rho, \phi)$ is a representation of $A$ if and only if there is a 3-Hom-Lie algebra structure (called the semi-direct product) on the direct sum $A \oplus V$ of vector spaces, defined by

$$
\begin{aligned}
{\left[x_{1}+v_{1}, x_{2}+v_{2}, x_{3}+v_{3}\right]_{A \oplus V} } & =\left[x_{1}, x_{2}, x_{3}\right]+\rho\left(x_{1}, x_{2}\right) v_{3}+\rho\left(x_{3}, x_{1}\right) v_{2}+\rho\left(x_{2}, x_{3}\right) v_{1} \\
\alpha_{A \oplus V}\left(x_{1}+v_{1}\right) & =\alpha\left(x_{1}\right)+\phi\left(v_{1}\right)
\end{aligned}
$$

for $x_{i} \in A, v_{i} \in V, 1 \leq i \leq 3$. We denote this semi-direct product 3-Hom-Lie algebra by $A \ltimes_{\rho} V$.

Proposition 1.2. Let $(V, \rho, \phi)$ be a representation of a 3-Hom-Lie algebra A. Then the following identities hold:

$$
\begin{aligned}
& \left(\rho\left(\left[x_{1}, x_{2}, x_{3}\right], \alpha\left(x_{4}\right)\right)-\rho\left(\left[x_{1}, x_{2}, x_{4}\right], \alpha\left(x_{3}\right)\right]\right) \\
& \left.+\rho\left(\left[x_{1}, x_{3}, x_{4}\right], \alpha\left(x_{2}\right)\right)-\rho\left(\left[x_{2}, x_{3}, x_{4}\right], \alpha\left(x_{1}\right)\right)\right) \circ \phi=0 \\
& \rho\left(\alpha\left(x_{1}\right), \alpha\left(x_{2}\right)\right) \rho\left(x_{3}, x_{4}\right)+\rho\left(\alpha\left(x_{2}\right), \alpha\left(x_{3}\right)\right) \rho\left(x_{1}, x_{4}\right)+\rho\left(\alpha\left(x_{3}\right), \alpha\left(x_{1}\right)\right) \rho\left(x_{2}, x_{4}\right) \\
& +\rho\left(\alpha\left(x_{3}\right), \alpha\left(x_{4}\right)\right) \rho\left(x_{1}, x_{2}\right)+\rho\left(\alpha\left(x_{1}\right), \alpha\left(x_{4}\right)\right) \rho\left(x_{2}, x_{3}\right)+\rho\left(\alpha\left(x_{2}\right), \alpha\left(x_{4}\right)\right) \rho\left(x_{3}, x_{1}\right)=0
\end{aligned}
$$

for $x_{i} \in A, 1 \leq i \leq 4$.

Let $(V, \rho, \phi)$ be a representation of a 3-Hom-Lie algebra $(A,[\cdot, \cdot \cdot \cdot], \alpha)$. In the sequel, we always assume that $\phi$ is invertible. Define $\rho^{*}: A \wedge A \longrightarrow g l\left(V^{*}\right)$ as usual by

$$
\left\langle\rho^{*}(x, y)(\xi), u\right\rangle=-\langle\xi, \rho(x, y)(u)\rangle, \quad \forall x, y \in A, u \in V, \xi \in V^{*} .
$$

However, in general $\rho^{*}$ is not a representation of $A$ anymore. To our knowledge, people need to add a very strong condition to obtain a representation on the dual space in the former study. We recall the dual representation of a representation of a 3-Hom-Lie algebra without any additional condition.

Define $\rho^{\star}: A \wedge A \longrightarrow g l\left(V^{*}\right)$ by

$$
\rho^{\star}(x, y)(\xi):=\rho^{*}(\alpha(x), \alpha(y))\left(\left(\phi^{-2}\right)^{*}(\xi)\right), \quad \forall x, y \in A, \xi \in V^{*} .
$$

More precisely, we have

$$
\left\langle\rho^{\star}(x, y)(\xi), u\right\rangle=-\left\langle\xi, \rho\left(\alpha^{-1}(x), \alpha^{-1}(y)\right)\left(\phi^{-2}(u)\right)\right\rangle, \quad \forall x, y \in A, u \in V, \xi \in V^{*} .
$$

Lemma 1.3. Let $(V, \rho, \phi)$ be a representation of a 3 -Hom-Lie algebra $(A,[\cdot, \cdot, \cdot], \alpha)$. Then $\rho^{\star}: A \longrightarrow g l\left(V^{*}\right)$ defined above by (1.10) is a representation of $(A,[\cdot, \cdot, \cdot], \alpha)$ on $V^{*}$ with respect to $\left(\phi^{-1}\right)^{*}$.

Proof. For any $x, y \in A, \xi \in V^{*}$ and $u \in V$, we have

$$
\begin{aligned}
\left\langle\rho^{\star}(\alpha(x), \alpha(y))\left(\left(\phi^{-1}\right)^{*}(\xi)\right), u\right\rangle & =-\left\langle\left(\phi^{-1}\right)^{*}(\xi), \rho(x, y) \phi^{-2}(u)\right\rangle \\
& =-\left\langle\xi, \rho\left(\alpha^{-1}(x), \alpha^{-1}(y)\right) \phi^{-3}(u)\right\rangle
\end{aligned}
$$

and

$$
\begin{aligned}
\left\langle\left(\phi^{-1}\right)^{*} \rho^{\star}(x, y)(\xi), u\right\rangle & =\left\langle\rho^{\star}(x, y)(\xi), \phi^{-1}(u)\right\rangle \\
& =-\left\langle\xi, \rho\left(\alpha^{-1}(x), \alpha^{-1}(y)\right) \phi^{-3}(u)\right\rangle,
\end{aligned}
$$

which implies that $\left(\phi^{-1}\right)^{*} \rho^{\star}(x, y)=\rho^{\star}(\alpha(x), \alpha(y))\left(\phi^{-1}\right)^{*}$. On the other hand,

$$
\left\langle\rho^{\star}\left(\alpha\left(x_{1}\right), \alpha\left(x_{2}\right)\right) \rho^{\star}\left(x_{3}, x_{4}\right)(\xi), u\right\rangle-\left\langle\rho^{\star}\left(\alpha\left(x_{3}\right), \alpha\left(x_{4}\right)\right) \rho^{\star}\left(x_{1}, x_{2}\right)(\xi), u\right\rangle
$$




$$
\begin{aligned}
& =\left\langle\xi, \rho\left(\alpha^{-1}\left(x_{3}\right), \alpha^{-1}\left(x_{4}\right)\right) \rho\left(\alpha^{-2}\left(x_{1}\right), \alpha^{-2}\left(x_{2}\right)\right) \phi^{-4}(u)\right\rangle \\
& -\left\langle\xi, \rho\left(\alpha^{-1}\left(x_{1}\right), \alpha^{-1}\left(x_{2}\right)\right) \rho\left(\alpha^{-2}\left(x_{3}\right), \alpha^{-2}\left(x_{4}\right)\right) \phi^{-4}(u)\right\rangle, \\
\left\langle\rho^{\star}\left(\left[x_{1}, x_{2}, x_{3}\right], \alpha\left(x_{4}\right)\right)\left(\phi^{-1}\right)^{*}(\xi), u\right\rangle-\left\langle\rho^{\star}\left(\left[x_{1}, x_{2}, x_{4}\right], \alpha\left(x_{3}\right)\right)\left(\phi^{-1}\right)^{*}(\xi), u\right\rangle & =-\left\langle\xi, \rho\left(\left[\alpha^{-2}\left(x_{1}\right), \alpha^{-2}\left(x_{2}\right), \alpha^{-2}\left(x_{3}\right)\right], \alpha^{-1}\left(x_{4}\right)\right) \phi^{-3}(u)\right\rangle \\
+ & \left\langle\xi, \rho\left(\left[\alpha^{-2}\left(x_{1}\right), \alpha^{-2}\left(x_{2}\right), \alpha^{-2}\left(x_{4}\right)\right], \alpha^{-1}\left(x_{3}\right)\right) \phi^{-3}(u)\right\rangle .
\end{aligned}
$$

Since $(\rho, \phi)$ is a representation, then we obtain

$$
\begin{aligned}
& \rho^{\star}\left(\alpha\left(x_{1}\right), \alpha\left(x_{2}\right)\right) \rho^{\star}\left(x_{3}, x_{4}\right)-\rho^{\star}\left(\alpha\left(x_{3}\right), \alpha\left(x_{4}\right)\right) \rho^{\star}\left(x_{1}, x_{2}\right) \\
= & \rho^{\star}\left(\left[x_{1}, x_{2}, x_{3}\right], \alpha\left(x_{4}\right)\right)\left(\phi^{-1}\right)^{\star}-\rho^{\star}\left(\left[x_{1}, x_{2}, x_{4}\right], \alpha\left(x_{3}\right)\right)\left(\phi^{-1}\right)^{\star} .
\end{aligned}
$$

The identity (1.5) can be shown similarly. Therefore $\rho^{\star}$ is a representation of $A$ on $V^{*}$ with respect to $\left(\phi^{-1}\right)^{*}$.

The tuple $\left(V^{*}, \rho^{\star},\left(\phi^{-1}\right)^{*}\right)$ is called the dual representation of $(V, \rho, \phi)$ for the 3-Hom-Lie algebra $(A,[\cdot, \cdot, \cdot], \alpha)$.

Corollary 1.4. Let $(A,[\cdot, \cdot, \cdot], \alpha)$ be a 3-Hom-Lie algebra. Then $a d^{\star}: A \rightarrow \operatorname{gl}\left(A^{*}\right)$ defined by

$$
\left\langle a d^{\star}(x, y)(\xi), z\right\rangle=-\left\langle\xi, a d\left(\alpha^{-1}(x), \alpha^{-1}(y)\right)\left(\alpha^{-2}(z)\right)\right\rangle, \quad \forall x, y, z \in A, \xi \in A^{*}
$$

is a representation of $(A,[\cdot, \cdot, \cdot], \alpha)$ on $A^{*}$ with respect to $\left(\alpha^{-1}\right)^{*}$, which is called the coadjoint representation.

In the next section, we recall the definition of 3-Hom-pre-Lie algebras and give construction result in terms of $O$-operators on 3-Hom-Lie algebras (see [27]). The notion of $O$-operator is useful tool for the construction of the solutions of the classical Yang-Baxter equation. Let $(A,[\cdot, \cdot, \cdot], \alpha)$ be a 3-Hom-Lie algebra and $(V, \rho, \phi)$ a representation. A linear operator $T: V \rightarrow A$ is called an $O$-operator associated to $(V, \rho, \phi)$ if $T$ satisfies

$$
\begin{aligned}
{[T u, T v, T w] } & =T(\rho(T u, T v) w+\rho(T v, T w) u+\rho(T w, T u) v), \quad \forall u, v, w \in V, \\
T \phi & =\alpha T .
\end{aligned}
$$

A linear map $\mathcal{R}: A \rightarrow A$ is called a Rota-Baxter operator if its an $O$-operator associated to the adjoint representation. That is $\mathcal{R} \alpha=\alpha R$ and for any $x, y, z \in A$ we have

$$
[\mathcal{R}(x), \mathcal{R}(y), \mathcal{R}(z)]=\mathcal{R}([\mathcal{R}(x), \mathcal{R}(y), z]+[\mathcal{R}(x), y, \mathcal{R}(z)]+[x, \mathcal{R}(y), \mathcal{R}(z)]) .
$$

\section{Representations of 3-Hom-pre-Lie algebras}

In this section, we introduce the notion of a representation of a 3-Hom-pre-Lie algebra, construct the corresponding semidirect product 3-Hom-Lie algebra and give the dual representation of a given representation without any additional condition.

Definition 2.1. ([27]) Let $A$ be a vector space with a linear map $\{\cdot, \cdot, \cdot\}: A \otimes A \otimes A \rightarrow A$ and $\alpha: A \rightarrow A$ is a linear map. The triplet $(A,\{\cdot, \cdot, \cdot\}, \alpha)$ is called a 3-Hom-pre-Lie algebra if the following identities hold:

$$
\{x, y, z\}=-\{y, x, z\},
$$




$$
\begin{aligned}
\left\{\alpha\left(x_{1}\right), \alpha\left(x_{2}\right),\left\{x_{3}, x_{4}, x_{5}\right\}\right\}= & \left\{\left[x_{1}, x_{2}, x_{3}\right]^{C}, \alpha\left(x_{4}\right), \alpha\left(x_{5}\right)\right\}+\left\{\alpha\left(x_{3}\right),\left[x_{1}, x_{2}, x_{4}\right]^{C}, \alpha\left(x_{5}\right)\right\} \\
& +\left\{\alpha\left(x_{3}\right), \alpha\left(x_{4}\right),\left\{x_{1}, x_{2}, x_{5}\right\}\right\} \\
\left\{\left[x_{1}, x_{2}, x_{3}\right]^{C}, \alpha\left(x_{4}\right), \alpha\left(x_{5}\right)\right\}= & \left\{\alpha\left(x_{1}\right), \alpha\left(x_{2}\right),\left\{x_{3}, x_{4}, x_{5}\right\}\right\}+\left\{\alpha\left(x_{2}\right), \alpha\left(x_{3}\right),\left\{x_{1}, x_{4}, x_{5}\right\}\right\} \\
& +\left\{\alpha\left(x_{3}\right), \alpha\left(x_{1}\right),\left\{x_{2}, x_{4}, x_{5}\right\}\right\},
\end{aligned}
$$

where $x, y, z, x_{i} \in A, 1 \leq i \leq 5$ and $[\cdot, \cdot, \cdot]^{C}$ is defined by

$$
[x, y, z]^{C}=\{x, y, z\}+\{y, z, x\}+\{z, x, y\}, \quad \forall x, y, z \in A .
$$

Proposition 2.1. ([27]) Let $(A,\{\cdot, \cdot, \cdot\}, \alpha)$ be a 3-Hom-pre-Lie algebra. Then the induced 3-commutator given by Eq. (2.18) defines a 3-Hom-Lie algebra. It is called the sub-adjacent 3-Hom-Lie algebra of $(A,\{\cdot, \cdot, \cdot\}, \alpha)$ and $(A,\{\cdot, \cdot, \cdot\}, \alpha)$ is the compatible 3 -Hom-pre-Lie algebra of $\left(A,[\cdot, \cdot, \cdot]^{C}, \alpha\right)$.

The sub-adjacent 3-Hom-Lie algebra $(A,\{\cdot, \cdot, \cdot\}, \alpha)$ will be denoted by $A^{c}$.

Let $(A,\{r, \cdot, \cdot\}, \alpha)$ be a 3-Hom-pre-Lie algebra. Define the left multiplication $L: \wedge^{2} A \longrightarrow g l(A)$ by $L(x, y) z=\{x, y, z\}$ for all $x, y, z \in A$. Then $(A, L, \alpha)$ is a representation of the 3-Hom-Lie algebra $A^{c}$. Moreover, we define the right multiplication $R: \otimes^{2} A \rightarrow g l(A)$ by $R(x, y) z=\{z, x, y\}$.

By the definition of 3-Hom-pre-Lie algebra and the representation of a 3-Hom-Lie algebra, we immediately obtain

Proposition 2.2. If $A$ is a vector space with linear maps $\{\cdot, \cdot, \cdot\}: A \otimes A \otimes A \rightarrow A$ and $\alpha: A \rightarrow A$ satisfying Eq. (2.16). Then $(A,\{\cdot, \cdot, \cdot\}, \alpha)$ is a 3-Hom-pre-Lie algebra if $\left(A,[\cdot, \cdot, \cdot]^{C}, \alpha\right)$ is a 3-Hom-Lie algebra and $(A, L, \alpha)$ gives a representation of it.

New identities of 3-Hom-pre-Lie algebras can be derived from Proposition 1.2. For example,

Corollary 2.3. Let $(A,\{\cdot, \cdot, \cdot\}, \alpha)$ be a 3-Hom-pre-Lie algebra. Then the following identities hold:

$$
\begin{aligned}
& \left\{\left[x_{1}, x_{2}, x_{3}\right]^{C}, \alpha\left(x_{4}\right), \alpha\left(x_{5}\right)\right\}-\left\{\left[x_{1}, x_{2}, x_{4}\right]^{C}, \alpha\left(x_{3}\right), \alpha\left(x_{5}\right)\right\} \\
+ & \left\{\left[x_{1}, x_{3}, x_{4}\right]^{C}, \alpha\left(x_{2}\right), \alpha\left(x_{5}\right)\right\}-\left\{\left[x_{2}, x_{3}, x_{4}\right]^{C}, \alpha\left(x_{1}\right), \alpha\left(x_{5}\right)\right\}=0, \\
& \left\{\alpha\left(x_{1}\right), \alpha\left(x_{2}\right),\left\{x_{3}, x_{4}, x_{5}\right\}\right\}+\left\{\alpha\left(x_{3}\right), \alpha\left(x_{4}\right),\left\{x_{1}, x_{2}, x_{5}\right\}\right\}+\left\{\alpha\left(x_{2}\right), \alpha\left(x_{4}\right),\left\{x_{3}, x_{1}, x_{5}\right\}\right\} \\
+ & \left\{\alpha\left(x_{3}\right), \alpha\left(x_{1}\right),\left\{x_{2}, x_{4}, x_{5}\right\}\right\}+\left\{\alpha\left(x_{2}\right), \alpha\left(x_{3}\right),\left\{x_{1}, x_{4}, x_{5}\right\}\right\}+\left\{\alpha\left(x_{1}\right), \alpha\left(x_{4}\right),\left\{x_{2}, x_{3}, x_{5}\right\}\right\}=0,
\end{aligned}
$$

for $x_{i} \in A, 1 \leq i \leq 5$.

Proposition 2.4. ([27]) Let $(A,[\cdot, \cdot, \cdot], \alpha)$ be a 3-Hom-Lie algebra and $(V, \rho, \phi)$ a representation. Suppose that the linear map $T: V \rightarrow A$ is an $O$-operator associated to $(V, \rho, \phi)$. Then $(V,\{\cdot, \cdot, \cdot\}, \phi)$ is a 3-Hom-pre-Lie algebra where the bracket $\{\cdot, \cdot, \cdot\}$ is given by

$$
\{u, v, w\}=\rho(T u, T v) w, \quad \forall u, v, w \in V .
$$

Corollary 2.5. With the above conditions, $\left(V,[\cdot, \cdot, \cdot]^{C}, \phi\right)$ is a 3-Hom-Lie algebra as the sub-adjacent 3-HomLie algebra of the 3-Hom-pre-Lie algebra given in Proposition 2.4 and $T$ is a 3-Hom-Lie algebra morphism from $\left(V,[\cdot, \cdot, \cdot]^{C}, \phi\right)$ to $(A,[\cdot, \cdot, \cdot], \alpha)$. Furthermore, $T(V)=\{T v \mid v \in V\} \subset A$ is a 3-Hom-Lie subalgebra of $A$ and there is an induced 3-Hom-pre-Lie algebra structure $\{\cdot, \cdot, \cdot\}_{T(V)}$ on $T(V)$ given by

$$
\{T u, T v, T w\}_{T(V)}:=T\{u, v, w\}, \quad \forall u, v, w \in V .
$$

Corollary 2.6. Let $(A,[\cdot, \cdot, \cdot], \alpha)$ be a 3-Hom-Lie algebra. Then there exists a compatible 3-Hom-pre-Lie algebra if and only if there exists an invertible $O$-operator on $A$. 
Now, we introduce the definition of representation of 3-Hom-pre-Lie algebra and give some related results.

Definition 2.2. A representation of a 3-Hom-pre-Lie algebra $(A,\{\cdot, \cdot, \cdot\}, \alpha)$ on a vector space $V$ consists of a triplet $(l, r, \phi)$, where $l: \wedge^{2} A \rightarrow g l(V)$ is a representation of the sub-adjacent 3-HomLie algebra $A^{c}$ on $V$ with respect to $\phi \in E n d(V)$ and $r: \otimes^{2} A \rightarrow g l(V)$ is a linear map such that for all $x_{1}, x_{2}, x_{3}, x_{4} \in A$, the following conditions hold

$$
\begin{aligned}
& \phi \circ r\left(x_{1}, x_{2}\right)=r\left(\alpha\left(x_{1}\right), \alpha\left(x_{2}\right)\right) \circ \phi, \\
& l\left(\alpha\left(x_{1}\right), \alpha\left(x_{2}\right)\right) r\left(x_{3}, x_{4}\right)=r\left(\alpha\left(x_{3}\right), \alpha\left(x_{4}\right)\right) \mu\left(x_{1}, x_{2}\right)+r\left(\left[x_{1}, x_{2}, x_{3}\right]^{C}, \alpha\left(x_{4}\right)\right) \circ \phi+r\left(\alpha\left(x_{3}\right),\left\{x_{1}, x_{2}, x_{4}\right\}\right) \circ \phi, \\
& r\left(\left[x_{1}, x_{2}, x_{3}\right]^{C}, \alpha\left(x_{4}\right)\right) \circ \phi=l\left(\alpha\left(x_{1}\right), \alpha\left(x_{2}\right)\right) r\left(x_{3}, x_{4}\right)+l\left(\alpha\left(x_{2}\right), \alpha\left(x_{3}\right)\right) r\left(x_{1}, x_{4}\right)+l\left(\alpha\left(x_{3}\right), \alpha\left(x_{1}\right)\right) r\left(x_{2}, x_{4}\right), \\
& r\left(\alpha\left(x_{1}\right),\left\{x_{2}, x_{3}, x_{4}\right\}\right) \circ \phi=r\left(\alpha\left(x_{3}\right), \alpha\left(x_{4}\right)\right) \mu\left(x_{1}, x_{2}\right)-r\left(\alpha\left(x_{2}\right), \alpha\left(x_{4}\right)\right) \mu\left(x_{1}, x_{3}\right)+l\left(\alpha\left(x_{2}\right), \alpha\left(x_{3}\right)\right) r\left(x_{1}, x_{4}\right), \\
& r\left(\alpha\left(x_{3}\right), \alpha\left(x_{4}\right)\right) \mu\left(x_{1}, x_{2}\right)=l\left(\alpha\left(x_{1}\right), \alpha\left(x_{2}\right)\right) r\left(x_{3}, x_{4}\right)-r\left(\alpha\left(x_{2}\right),\left\{x_{1}, x_{3}, x_{4}\right\}\right) \circ \phi+r\left(\alpha\left(x_{1}\right),\left\{x_{2}, x_{3}, x_{4}\right\}\right) \circ \phi,
\end{aligned}
$$

where $\mu(x, y)=l(x, y)+r(x, y)-r(y, x)$, for any $x, y \in A$.

It is obvious that $(A, L, R, \alpha)$ is a representation of a 3-Hom-pre-Lie algebra on itself, which is called the adjoint representation.

Theorem 2.7. Let $(A,\{\cdot, \cdot, \cdot\})$ be 3-pre-Lie algebras and $(V, l, r)$ be a representation on $A$. Let $\phi: V \rightarrow V$ and $\alpha: A \rightarrow A$ be two morphisms such that:

$$
\phi \circ l\left(x_{1}, x_{2}\right)=l\left(\alpha\left(x_{1}\right), \alpha\left(x_{2}\right)\right) \circ \phi, \quad \phi \circ r\left(x_{1}, x_{2}\right)=r\left(\alpha\left(x_{1}\right), \alpha\left(x_{2}\right)\right) \circ \phi, \forall x_{1}, x_{2} \in A .
$$

Then $(V, \tilde{l}, \tilde{r}, \phi)$ is a representation of 3 -Hom-pre-Lie algebras $\left(A,\{\cdot, \cdot, \cdot\}_{\alpha, C}=\alpha \circ\{\cdot, \cdot, \cdot\}^{C}, \alpha\right)$, where $\tilde{l}=\phi \circ l$ and $\tilde{r}=\phi \circ r$.

Proof. We will proof the equality (2.25), i.e. for all $x_{1}, x_{2}, x_{3}, x_{4} \in A$

$\tilde{r}\left(\left[x_{1}, x_{2}, x_{3}\right]_{\alpha, C}, \alpha\left(x_{4}\right)\right) \circ \phi-\tilde{l}\left(\alpha\left(x_{1}\right), \alpha\left(x_{2}\right)\right) \tilde{r}\left(x_{3}, x_{4}\right)-\tilde{l}\left(\alpha\left(x_{2}\right), \alpha\left(x_{3}\right)\right) \tilde{r}\left(x_{1}, x_{4}\right)-\tilde{l}\left(\alpha\left(x_{3}\right), \alpha\left(x_{1}\right)\right) \tilde{r}\left(x_{2}, x_{4}\right)=0$.

We have

$$
\begin{aligned}
& \tilde{r}\left(\left[x_{1}, x_{2}, x_{3}\right]_{\alpha, C}, \alpha\left(x_{4}\right)\right) \circ \phi-\tilde{l}\left(\alpha\left(x_{1}\right), \alpha\left(x_{2}\right)\right) \tilde{r}\left(x_{3}, x_{4}\right)-\tilde{l}\left(\alpha\left(x_{2}\right), \alpha\left(x_{3}\right)\right) \tilde{r}\left(x_{1}, x_{4}\right)-\tilde{l}\left(\alpha\left(x_{3}\right), \alpha\left(x_{1}\right)\right) \tilde{r}\left(x_{2}, x_{4}\right) \\
= & \phi \circ r\left(\alpha \circ\left[x_{1}, x_{2}, x_{3}\right], \alpha\left(x_{4}\right)\right) \circ \phi-\phi \circ l\left(\alpha\left(x_{1}\right), \alpha\left(x_{2}\right)\right) \phi \circ r\left(x_{3}, x_{4}\right) \\
- & \phi \circ l\left(\alpha\left(x_{2}\right), \alpha\left(x_{3}\right)\right) \phi \circ r\left(x_{1}, x_{4}\right)-\phi \circ l\left(\alpha\left(x_{3}\right), \alpha\left(x_{1}\right)\right) \phi \circ r\left(x_{2}, x_{4}\right) \\
= & \phi^{2} \circ\left(r\left(\left[x_{1}, x_{2}, x_{3}\right], x_{4}\right)-l\left(x_{1}, x_{2}\right) r\left(x_{3}, x_{4}\right)-l\left(x_{2}, x_{3}\right) r\left(x_{1}, x_{4}\right)-l\left(x_{3}, x_{1}\right) r\left(x_{2}, x_{4}\right)\right)=0 .
\end{aligned}
$$

The other identities of the definition (2.2) can be shown similarly.

Proposition 2.8. Let $(A,\{\cdot, \cdot, \cdot\}, \alpha)$ be a 3-Hom-pre-Lie algebra, $V$ a vector space and $l, r: \otimes^{2} A \rightarrow g l(V)$ two linear maps and $\phi: V \rightarrow V$ a morphism algebra. Then $(V, l, r, \phi)$ is a representation of $A$ if and only if there is a 3-Hom-pre-Lie algebra structure (called the semi-direct product) on the direct sum $A \oplus V$ of vector spaces, defined by

$$
\begin{aligned}
(\alpha+\phi)_{A \oplus V}\left(x_{1}+u_{1}\right) & =\alpha\left(x_{1}\right)+\phi\left(u_{1}\right), \\
{\left[x_{1}+u_{1}, x_{2}+u_{2}, x_{3}+u_{3}\right]_{A \oplus V} } & =\left\{x_{1}, x_{2}, x_{3}\right\}+l\left(x_{1}, x_{2}\right) u_{3}-r\left(x_{1}, x_{3}\right) u_{2}+r\left(x_{2}, x_{3}\right) u_{1},
\end{aligned}
$$

for $x_{i} \in A, u_{i} \in V, 1 \leq i \leq 3$. We denote this semi-direct product 3-Hom-pre-Lie algebra by $A \ltimes_{l, r}^{\alpha, \phi} V$. 
Proof. Let $x_{i} \in A$ and $u_{i} \in V$, for $1 \leq i \leq 5$. Then

$$
\begin{aligned}
& {\left[(\alpha+\phi)_{A \oplus V}\left(x_{1}+u_{1}\right),(\alpha+\phi)_{A \oplus V}\left(x_{2}+u_{2}\right),\left[x_{3}+u_{3}, x_{4}+u_{4}, x_{5}+u_{5}\right]_{A \oplus V}\right]_{A \oplus V} } \\
- & {\left[(\alpha+\phi)_{A \oplus V}\left(x_{3}+u_{3}\right),(\alpha+\phi)_{A \oplus V}\left(x_{4}+u_{4}\right),\left[x_{1}+u_{1}, x_{2}+u_{2}, x_{5}+u_{5}\right]_{A \oplus V}\right]_{A \oplus V} } \\
- & {\left[\left[x_{1}+u_{1}, x_{2}+u_{2}, x_{3}+u_{3}\right]_{A \oplus V^{\prime}}^{C}(\alpha+\phi)_{A \oplus V}\left(x_{4}+u_{4}\right),(\alpha+\phi)_{A \oplus V}\left(x_{5}+u_{5}\right)\right]_{A \oplus V} } \\
+ & {\left[\left[x_{1}+u_{1}, x_{2}+u_{2}, x_{4}+u_{4}\right]_{A \oplus V^{\prime}}^{C}(\alpha+\phi)_{A \oplus V}\left(x_{3}+u_{3}\right),(\alpha+\phi)_{A \oplus V}\left(x_{5}+u_{5}\right)\right]_{A \oplus V} } \\
= & \left\{\alpha\left(x_{1}\right), \alpha\left(x_{2}\right),\left\{x_{3}, x_{4}, x_{5}\right\}\right\}+l\left(\alpha\left(x_{1}\right), \alpha\left(x_{2}\right)\right) l\left(x_{3}, x_{4}\right) \phi\left(u_{5}\right)-l\left(\alpha\left(x_{1}\right), \alpha\left(x_{2}\right)\right) r\left(x_{3}, x_{5}\right) \phi\left(u_{4}\right) \\
+ & l\left(\alpha\left(x_{1}\right), \alpha\left(x_{2}\right)\right) r\left(x_{4}, x_{5}\right) \phi\left(u_{3}\right)-r\left(\alpha\left(x_{1}\right),\left\{x_{3}, x_{4}, x_{5}\right\}\right) \phi\left(u_{2}\right)+r\left(\alpha\left(x_{2}\right),\left\{x_{3}, x_{4}, x_{5}\right\}\right) \phi\left(u_{1}\right) \\
- & \left\{\alpha\left(x_{3}\right), \alpha\left(x_{4}\right),\left\{x_{1}, x_{2}, x_{5}\right\}\right\}-l\left(\alpha\left(x_{3}\right), \alpha\left(x_{4}\right)\right) l\left(x_{1}, x_{3}\right) \phi\left(u_{5}\right)+l\left(\alpha\left(x_{3}\right), \alpha\left(x_{4}\right)\right) r\left(x_{1}, x_{5}\right) \phi\left(u_{2}\right) \\
- & l\left(\alpha\left(x_{3}\right), \alpha\left(x_{4}\right)\right) r\left(x_{2}, x_{5}\right) \phi\left(u_{1}\right)+r\left(\alpha\left(x_{3}\right),\left\{x_{1}, x_{2}, x_{5}\right\}\right) \phi\left(u_{4}\right)-r\left(\alpha\left(x_{4}\right),\left\{x_{1}, x_{2}, x_{5}\right\}\right) \phi\left(u_{3}\right) \\
- & \left\{\left[x_{1}, x_{2}, x_{3}\right]^{C}, \alpha\left(x_{4}\right), \alpha\left(x_{5}\right)\right\}+l\left(\left[x_{1}, x_{2}, x_{3}\right]^{C}, \alpha\left(x_{4}\right)\right) \phi\left(u_{5}\right)-l\left(\left[x_{1}, x_{2}, x_{3}\right]^{C}, \alpha\left(x_{5}\right)\right) \phi\left(u_{4}\right) \\
+ & \cup_{1,2,3}\left(r\left(\alpha\left(x_{4}\right), \alpha\left(x_{5}\right)\right) l\left(x_{1}, x_{2}\right) \phi\left(u_{3}\right)-r\left(\alpha\left(x_{4}\right), \alpha\left(x_{5}\right)\right) r\left(x_{1}, x_{3}\right) \phi\left(u_{2}\right)+r\left(\alpha\left(x_{4}\right), \alpha\left(x_{5}\right)\right) r\left(x_{2}, x_{3}\right) \phi\left(u_{1}\right)\right) \\
+ & \left\{\left[x_{1}, x_{2}, x_{4}\right]^{C}, \alpha\left(x_{3}\right), \alpha\left(x_{5}\right)\right\}-l\left(\left[x_{1}, x_{2}, x_{4}\right]^{C}, \alpha\left(x_{3}\right)\right) \phi\left(u_{5}\right)+l\left(\left[x_{1}, x_{2}, x_{4}\right]^{C}, \alpha\left(x_{5}\right)\right) \phi\left(u_{3}\right) \\
- & \cup_{1,2,4}\left(r\left(\alpha\left(x_{3}\right), \alpha\left(x_{5}\right)\right) l\left(x_{1}, x_{2}\right) \phi\left(u_{4}\right)+r\left(\alpha\left(x_{3}\right), \alpha\left(x_{5}\right)\right) r\left(x_{1}, x_{4}\right) \phi\left(u_{2}\right)-r\left(\alpha\left(x_{3}\right), \alpha\left(x_{5}\right)\right) r\left(x_{2}, x_{4}\right) \phi\left(u_{1}\right)\right)=0
\end{aligned}
$$

if and only if the identities (2.24)-(2.26) hold. Moreover,

$$
\begin{aligned}
& {\left[\left[x_{1}+u_{1}, x_{2}+u_{2}, x_{3}+u_{3}\right]_{A \oplus V}^{C}(\alpha+\phi)_{A \oplus V}\left(x_{4}+u_{4}\right),(\alpha+\phi)_{A \oplus V}\left(x_{5}+u_{5}\right)\right]_{A \oplus V} } \\
- & {\left[(\alpha+\phi)_{A \oplus V}\left(x_{1}+u_{1}\right),(\alpha+\phi)_{A \oplus V}\left(x_{2}+u_{2}\right),\left[x_{3}+u_{3}, x_{4}+u_{4}, x_{5}+u_{5}\right]_{A \oplus V}\right]_{A \oplus V} } \\
- & {\left[(\alpha+\phi)_{A \oplus V}\left(x_{2}+u_{2}\right),(\alpha+\phi)_{A \oplus V}\left(x_{3}+u_{3}\right),\left[x_{1}+u_{1}, x_{4}+u_{4}, x_{5}+u_{5}\right]_{A \oplus V}\right]_{A \oplus V} } \\
- & {\left[(\alpha+\phi)_{A \oplus V}\left(x_{3}+u_{3}\right),(\alpha+\phi)_{A \oplus V}\left(x_{1}+u_{1}\right),\left[x_{2}+u_{2}, x_{4}+u_{4}, x_{5}+u_{5}\right]_{A \oplus V}\right]_{A \oplus V} } \\
= & {\left[\left[x_{1}+u_{1}, x_{2}+u_{2}, x_{3}+u_{3}\right]_{A \oplus V}^{C}(\alpha+\phi)_{A \oplus V}\left(x_{4}+u_{4}\right),(\alpha+\phi)_{A \oplus V}\left(x_{5}+u_{5}\right)\right]_{A \oplus V} } \\
- & -U_{1,2,3}\left[(\alpha+\phi)_{A \oplus V}\left(x_{1}+u_{1}\right),(\alpha+\phi)_{A \oplus V}\left(x_{2}+u_{2}\right),\left[x_{3}+u_{3}, x_{4}+u_{4}, x_{5}+u_{5}\right]_{A \oplus V}\right]_{A \oplus V} \\
= & \left\{\left[x_{1}, x_{2}, x_{3}\right]^{C}, \alpha\left(x_{4}\right), \alpha\left(x_{5}\right)\right\}-l\left(\left[x_{1}, x_{2}, x_{3}\right]^{C}, \alpha\left(x_{4}\right)\right) \phi\left(u_{5}\right)+l\left(\left[x_{1}, x_{2}, x_{3}\right]^{C}, \alpha\left(x_{5}\right)\right) \phi\left(u_{4}\right) \\
- & U_{1,2,3}\left(r\left(\alpha\left(x_{4}\right), \alpha\left(x_{5}\right)\right) l\left(x_{1}, x_{2}\right) \phi\left(u_{3}\right)-r\left(\alpha\left(x_{4}\right), \alpha\left(x_{5}\right)\right) r\left(x_{1}, x_{3}\right) \phi\left(u_{2}\right)+r\left(\alpha\left(x_{4}\right), \alpha\left(x_{5}\right)\right) r\left(x_{2}, x_{3}\right) \phi\left(u_{1}\right)\right) \\
- & \cup_{1,2,3}\left(\left\{\alpha\left(x_{1}\right), \alpha\left(x_{2}\right),\left\{x_{3}, x_{4}, x_{5}\right\}\right\}+l\left(\alpha\left(x_{1}\right), \alpha\left(x_{2}\right)\right) l\left(x_{3}, x_{4}\right) \phi\left(u_{5}\right)\right. \\
- & l\left(\alpha\left(x_{1}\right), \alpha\left(x_{2}\right)\right) r\left(x_{3}, x_{5}\right) \phi\left(u_{4}\right)+l\left(\alpha\left(x_{1}\right), \alpha\left(x_{2}\right)\right) r\left(x_{4}, x_{5}\right) \phi\left(u_{3}\right) \\
& \left.-r\left(\alpha\left(x_{1}\right),\left\{x_{3}, x_{4}, x_{5}\right\}\right) \phi\left(u_{2}\right)+r\left(\alpha\left(x_{2}\right),\left\{x_{3}, x_{4}, x_{5}\right\}\right) \phi\left(u_{1}\right)\right) .=0
\end{aligned}
$$

if and only if the identities (2.25)-(2.27) hold.

Proposition 2.9. Let $(V, l, r, \phi)$ be a representation of a 3 -Hom-pre-Lie algebra $(A,\{\cdot, \cdot, \cdot\}, \alpha)$. Then $\mu$ is a representation of the sub-adjacent 3-Hom-Lie algebra $\left(A^{c},[\cdot, \cdot, \cdot]^{C}, \alpha\right)$ on the vector space $V$ with respect to $\phi$, where $\mu$ is defined in definition 2.2

Proof. Straightforward.

Now, we study the dual representation of a 3-Hom-pre-Lie algebra. We give a definition without any additional condition, similarly as for Hom-Lie algebras and 3-Hom-Lie algebras. Let $(V, l, r, \phi)$ be a representation of a 3-Hom-pre-Lie algebra $(A,\{\cdot, \cdot, \cdot\}, \alpha)$. Define $l^{*}: A \wedge A \longrightarrow g l\left(V^{*}\right)$ and $r^{*}: A \otimes A \longrightarrow g l\left(V^{*}\right)$ as usual by

$$
\begin{aligned}
\left\langle l^{*}(x, y)(\xi), u\right\rangle & =-\langle\xi, l(x, y)(u)\rangle, \\
\left\langle r^{*}(x, y)(\xi), u\right\rangle & =-\langle\xi, r(x, y)(u)\rangle,
\end{aligned}
$$


for all $x, y \in A, u \in V, \xi \in V^{*}$. However, in general $\left(l^{*}-r^{*} \tau+r^{*},-r^{*}\right)$ is not a representation of $A$ anymore. We will try to construct the dual representation of a representation of a 3-Hom-pre-Lie algebra without any condition.

Define $l^{\star}: A \wedge A \longrightarrow g l\left(V^{*}\right)$ and $r^{\star}: A \otimes A \longrightarrow g l\left(V^{*}\right)$ by

$$
\begin{aligned}
& l^{\star}(x, y)(\xi):=l^{*}(\alpha(x), \alpha(y))\left(\left(\phi^{-2}\right)^{*}(\xi)\right), \\
& r^{\star}(x, y)(\xi):=r^{*}(\alpha(x), \alpha(y))\left(\left(\phi^{-2}\right)^{*}(\xi)\right) .
\end{aligned}
$$

More precisely, we have

$$
\begin{aligned}
& \left\langle l^{\star}(x, y)(\xi), u\right\rangle=-\left\langle\xi, l\left(\alpha^{-1}(x), \alpha^{-1}(y)\right)\left(\phi^{-2}(u)\right)\right\rangle, \\
& \left\langle r^{\star}(x, y)(\xi), u\right\rangle=-\left\langle\xi, r\left(\alpha^{-1}(x), \alpha^{-1}(y)\right)\left(\phi^{-2}(u)\right)\right\rangle,
\end{aligned}
$$

for any $x, y \in A, u \in V, \xi \in V^{*}$.

Proposition 2.10. Let $(V, l, r, \phi)$ be a representation of a 3-Hom-pre-Lie algebra $(A,\{\cdot, \cdot, \cdot\}, \alpha)$. Then $\left(\mu^{\star},-r^{\star}\right)$ is a representation of the 3-Hom-pre-Lie algebra $(A,\{\cdot, \cdot, \cdot\}, \alpha)$ on the vector space $V^{*}$ with respect to $\left(\phi^{-1}\right)^{*}$, which is called the dual representation of the representation $(V, l, r, \phi)$.

Proof. Straightforward.

If $(V, l, r, \phi)=(A, L, R, \alpha)$ is the adjoint representation of a 3-Hom-pre-Lie algebra $(A,\{\cdot, \cdot, \cdot\}, \alpha)$, then we obtain $\left(l^{\star}-r^{\star} \tau+r^{\star},-r^{\star}\right)=\left(a d^{\star},-R^{\star}\right)$ and $\left(\phi^{-1}\right)^{*}=\left(\alpha^{-1}\right)^{*}$.

Definition 2.3. Let $(V, l, r, \phi)$ be a representation of a 3-Hom-pre-Lie algebra $(A,\{\cdot, \cdot, \cdot\}, \alpha)$. A linear operator $T: V \rightarrow A$ is called and $O$-operator associated to $(V, l, r, \phi)$ if $T$ satisfies

$$
\begin{gathered}
T \phi=\alpha T . \\
\{T u, T v, T w\}=T(l(T u, T v) w-r(T u, T w) v+r(T v, T w) u), \quad \forall u, v, w \in V .
\end{gathered}
$$

If $V=A$, then $T$ is called a Rota-Baxter operator on $A$ of weight zero. That is

$$
\{\mathcal{R}(x), \mathcal{R}(y), \mathcal{R}(z)\}=\mathcal{R}(\{\mathcal{R}(x), \mathcal{R}(y), z\}+\{\mathcal{R}(x), y, \mathcal{R}(z)\}+\{x, \mathcal{R}(y), \mathcal{R}(z)\}),
$$

for all $x, y, z \in A$.

\section{3-Hom-L-dendriform algebras}

In this section, we introduce the notion of a 3-Hom-L-dendriform algebra which is exactly the ternary version of a Hom-L-dendriform algebra.

Definition 3.1. Let $A$ be a vector space with three linear maps $\nwarrow, \nearrow: \otimes^{3} A \rightarrow A$ and $\alpha: A \rightarrow A$. The 4-uple $(A, \nwarrow, \nearrow, \alpha)$ is called a 3-Hom-L-dendriform algebra if the following identities hold:

$$
\begin{aligned}
& \nwarrow\left(x_{1}, x_{2}, x_{3}\right)+\nwarrow\left(x_{2}, x_{1}, x_{3}\right)=0, \\
& \nwarrow\left(\alpha\left(x_{1}\right), \alpha\left(x_{2}\right), \nwarrow\left(x_{3}, x_{4}, x_{5}\right)\right)-\nwarrow\left(\alpha\left(x_{3}\right), \alpha\left(x_{4}\right), \nwarrow\left(x_{1}, x_{2}, x_{5}\right)\right) \\
& =\nwarrow\left(\left[x_{1}, x_{2}, x_{3}\right]^{C}, \alpha\left(x_{4}\right), \alpha\left(x_{5}\right)\right)-\nwarrow\left(\left[x_{1}, x_{2}, x_{4}\right]^{C}, \alpha\left(x_{3}\right), \alpha\left(x_{5}\right)\right), \\
& \nwarrow\left(\alpha\left(x_{1}\right), \alpha\left(x_{2}\right), \nearrow\left(x_{5}, x_{3}, x_{4}\right)\right)-\nearrow\left(\alpha\left(x_{5}\right), \alpha\left(x_{3}\right),\left\{x_{1}, x_{2}, x_{4}\right\}^{h}\right)
\end{aligned}
$$




$$
\begin{gathered}
=\nearrow\left(\alpha\left(x_{5}\right),\left[x_{1}, x_{2}, x_{3}\right]^{C}, \alpha\left(x_{4}\right)\right)+\nearrow\left(\left\{x_{1}, x_{2}, x_{5}\right\}^{v}, \alpha\left(x_{3}\right), \alpha\left(x_{4}\right)\right), \\
\nearrow\left(\alpha\left(x_{5}\right), \alpha\left(x_{1}\right),\left\{x_{2}, x_{3}, x_{4}\right\}^{h}\right)-\nwarrow\left(\alpha\left(x_{2}\right), \alpha\left(x_{3}\right), \nearrow\left(x_{5}, x_{1}, x_{4}\right)\right) \\
=\nearrow\left(\left\{x_{1}, x_{2}, x_{5}\right\}^{v}, \alpha\left(x_{3}\right), \alpha\left(x_{4}\right)\right)-\nearrow\left(\left\{x_{1}, x_{3}, x_{5}\right\}^{v}, \alpha\left(x_{2}\right), \alpha\left(x_{4}\right)\right), \\
\nwarrow\left(\left[x_{1}, x_{2}, x_{3}\right]^{C}, \alpha\left(x_{4}\right), \alpha\left(x_{5}\right)\right)=\cup_{1,2,3} \nwarrow\left(\alpha\left(x_{1}\right), \alpha\left(x_{2}\right), \nwarrow\left(x_{3}, x_{4}, x_{5}\right)\right), \\
\nearrow\left(\alpha\left(x_{5}\right),\left[x_{1}, x_{2}, x_{3}\right]^{C}, \alpha\left(x_{4}\right)\right)=\cup_{1,2,3} \nwarrow\left(\alpha\left(x_{1}\right), \alpha\left(x_{2}\right), \nearrow\left(x_{5}, x_{3}, x_{4}\right)\right), \\
\nwarrow\left(\alpha\left(x_{1}\right), \alpha\left(x_{2}\right), \nearrow\left(x_{5}, x_{3}, x_{4}\right)\right)+\nearrow\left(\alpha\left(x_{5}\right), \alpha\left(x_{1}\right),\left\{x_{2}, x_{3}, x_{4}\right\}^{h}\right) \\
=\nearrow\left(\alpha\left(x_{5}\right), \alpha\left(x_{2}\right),\left\{x_{1}, x_{3}, x_{4}\right\}^{h}\right)+\nearrow\left(\left\{x_{1}, x_{2}, x_{5}\right\}^{v}, \alpha\left(x_{3}\right), \alpha\left(x_{4}\right)\right),
\end{gathered}
$$

for all $x_{i} \in A, 1 \leq i \leq 5$, where

$$
\begin{aligned}
& \{x, y, z\}^{h}=\nwarrow(x, y, z)+\nearrow(x, y, z)-\nearrow(y, x, z), \\
& \{x, y, z\}^{v}=\nwarrow(x, y, z)+\nearrow(z, x, y)-\nearrow(z, y, x), \\
& {[x, y, z]^{C}=\bigcup_{x, y, z}\{x, y, z\}^{h}=\cup_{x, y, z}\{x, y, z\}^{v},}
\end{aligned}
$$

for an $x, y, z \in A$.

A morphism of 3-Hom-L-dendriform algebra $f:\left(A_{1}, \nwarrow_{1}, \nearrow_{1}, \alpha_{1}\right) \rightarrow\left(A_{2}, \nwarrow_{2}, \nearrow_{2}, \alpha_{2}\right)$ is a linear map such that:

$$
\begin{aligned}
f \nwarrow_{1}\left(x_{1}, x_{2}, x_{3}\right) & =\nwarrow_{2}\left(f\left(x_{1}\right), f\left(x_{2}\right), f\left(x_{3}\right)\right), \\
f \nearrow_{1}\left(x_{1}, x_{2}, x_{3}\right) & =\nearrow_{2}\left(f\left(x_{1}\right), f\left(x_{2}\right), f\left(x_{3}\right)\right), \\
f \circ \alpha_{1} & =\alpha_{2} \circ f,
\end{aligned}
$$

for all $x_{1}, x_{2}, x_{3} \in A_{1}$.

Proposition 3.1. Let $(A, \nwarrow, \nearrow)$ be a 3-L-dendriform algebra. Define two linear maps $\nwarrow_{\alpha}, \nearrow_{\alpha}: \otimes^{3} A \rightarrow A$ by

$$
\nwarrow_{\alpha}\left(x_{1}, x_{2}, x_{3}\right)=\nwarrow\left(\alpha\left(x_{1}\right), \alpha\left(x_{2}\right), \alpha\left(x_{3}\right)\right) ; \quad \nearrow_{\alpha}\left(x_{1}, x_{2}, x_{3}\right)=\nearrow\left(\alpha\left(x_{1}\right), \alpha\left(x_{2}\right), \alpha\left(x_{3}\right)\right), \forall x_{1}, x_{2}, x_{3} \in A .
$$

Then $\left(A, \nwarrow_{\alpha}, \nearrow_{\alpha}, \alpha\right)$ is 3-Hom-L-dendriform algebra.

Proof. For any $x_{1}, x_{2}, x_{3} \in A$, we have:

$$
\nwarrow_{\alpha}\left(x_{1}, x_{2}, x_{3}\right)+\nwarrow_{\alpha}\left(x_{2}, x_{1}, x_{3}\right)=\nwarrow\left(\alpha\left(x_{1}\right), \alpha\left(x_{2}\right), \alpha\left(x_{3}\right)\right)+\nwarrow\left(\alpha\left(x_{2}\right), \alpha\left(x_{1}\right), \alpha\left(x_{3}\right)\right)=0 .
$$

Moreover, we have for $x_{1}, x_{2}, x_{3}, x_{4}, x_{5} \in A$

$$
\begin{aligned}
& \nwarrow_{\alpha}\left(\alpha\left(x_{1}\right), \alpha\left(x_{2}\right), \nwarrow_{\alpha}\left(x_{3}, x_{4}, x_{5}\right)\right)-\nwarrow_{\alpha}\left(\alpha\left(x_{3}\right), \alpha\left(x_{4}\right), \nwarrow_{\alpha}\left(x_{1}, x_{2}, x_{5}\right)\right) \\
& =\nwarrow\left(\alpha^{2}\left(x_{1}\right), \alpha^{2}\left(x_{2}\right), \nwarrow\left(\alpha^{2}\left(x_{3}\right), \alpha^{2}\left(x_{4}\right), \alpha^{2}\left(x_{5}\right)\right)\right)-\nwarrow\left(\alpha^{2}\left(x_{3}\right), \alpha^{2}\left(x_{4}\right), \nwarrow\left(\alpha^{2}\left(x_{1}\right), \alpha^{2}\left(x_{2}\right), \alpha^{2}\left(x_{5}\right)\right)\right) \\
& =\nwarrow_{\alpha}\left(\left[x_{1}, x_{2}, x_{3}\right]^{\complement}, \alpha\left(x_{4}\right), \alpha\left(x_{5}\right)\right)-\nwarrow_{\alpha}\left(\left[x_{1}, x_{2}, x_{4}\right]^{\complement}, \alpha\left(x_{3}\right), \alpha\left(x_{5}\right)\right) .
\end{aligned}
$$

Which imply (3.36). Similarly, we obtain the identities (3.37)-(3.41).

Proposition 3.2. Let $(A, \nwarrow, \nearrow, \alpha)$ be a 3-Hom-L-dendriform algebra. The bracket defined in (3.42) (respectively (3.43) ) defines a 3-Hom-pre-Lie algebra structure on $A$ which is called the associated horizontal ( respectively associated vertical ) 3-Hom-pre-Lie algebra of $(A, \nwarrow, \nearrow, \alpha)$ and $(A, \nwarrow, \nearrow, \alpha)$ is also called a compatible 3-Hom-L-dendriform algebra structure on the 3-Hom-pre-Lie algebra $\left(A,\{\cdot, \cdot, \cdot\}^{h}, \alpha\right)$ ( respectively $\left.\left(A,\{\cdot, \cdot, \cdot\}^{v}, \alpha\right)\right)$. 
Proof. We will just prove that $\left(A,\{\cdot, \cdot, \cdot\}^{h}, \alpha\right)$ is a 3-Hom-pre-Lie algebra.

Note, first that $\{x, y, z\}^{h}=-\{y, x, z\}^{h}$, for any $x, y, z \in A$. Let $x_{i} \in A, 1 \leq i \leq 5$, then

$$
\begin{aligned}
& \left\{\alpha\left(x_{1}\right), \alpha\left(x_{2}\right),\left\{x_{3}, x_{4}, x_{5}\right\}^{h}\right\}^{h}-\left\{\alpha\left(x_{3}\right), \alpha\left(x_{4}\right),\left\{x_{1}, x_{2}, x_{5}\right\}^{h}\right\}^{h} \\
& -\left\{\left[x_{1}, x_{2}, x_{3}\right]^{C}, \alpha\left(x_{4}\right), \alpha\left(x_{5}\right)\right\}^{h}+\left\{\left[x_{1}, x_{2}, x_{4}\right]^{C}, \alpha\left(x_{3}\right), \alpha\left(x_{5}\right)\right\}^{h} \\
& =r_{1}+r_{2}+r_{3}+r_{4}+r_{5},
\end{aligned}
$$

where

$$
\begin{aligned}
r_{1} & =\nwarrow\left(\alpha\left(x_{1}\right), \alpha\left(x_{2}\right), \nwarrow\left(x_{3}, x_{4}, x_{5}\right)\right)-\nwarrow\left(\alpha\left(x_{3}\right), \alpha\left(x_{4}\right), \nwarrow\left(x_{1}, x_{2}, x_{5}\right)\right)-\nwarrow\left(\left[x_{1}, x_{2}, x_{3}\right]^{C}, \alpha\left(x_{4}\right), \alpha\left(x_{5}\right)\right) \\
& +\nwarrow\left(\left[x_{1}, x_{2}, x_{4}\right]^{C}, \alpha\left(x_{3}\right), \alpha\left(x_{5}\right)\right), \\
r_{2} & =\nearrow\left(\alpha\left(x_{3}\right),\left[x_{1}, x_{2}, x_{4}\right]^{C}, \alpha\left(x_{5}\right)\right)+\nearrow\left(\alpha\left(x_{3}\right), \alpha\left(x_{4}\right),\left\{x_{1}, x_{2}, x_{5}\right\}^{h}\right)-\nwarrow\left(\alpha\left(x_{1}\right), \alpha\left(x_{2}\right), \nearrow\left(x_{3}, x_{4}, x_{5}\right)\right) \\
& +\nearrow\left(\left\{x_{1}, x_{2}, x_{3}\right\}^{v}, \alpha\left(x_{4}\right), \alpha\left(x_{5}\right)\right), \\
r_{3} & =\nearrow\left(\alpha\left(x_{4}\right),\left[x_{1}, x_{2}, x_{3}\right]^{C}, \alpha\left(x_{5}\right)\right)+\nearrow\left(\alpha\left(x_{4}\right), \alpha\left(x_{3}\right),\left\{x_{1}, x_{2}, x_{5}\right\}^{h}\right)-\nwarrow\left(\alpha\left(x_{1}\right), \alpha\left(x_{2}\right), \nearrow\left(x_{4}, x_{3}, x_{5}\right)\right) \\
& +\nearrow\left(\left\{x_{1}, x_{2}, x_{4}\right\}^{v}, \alpha\left(x_{3}\right), \alpha\left(x_{5}\right)\right), \\
r_{4} & =\nearrow\left(\alpha\left(x_{1}\right), \alpha\left(x_{2}\right),\left\{x_{3}, x_{4}, x_{5}\right\}^{h}\right)-\nwarrow\left(\alpha\left(x_{3}\right), \alpha\left(x_{4}\right), \nearrow\left(x_{1}, x_{2}, x_{5}\right)\right)-\nearrow\left(\left\{x_{2}, x_{3}, x_{1}\right\}^{v}, \alpha\left(x_{4}\right), \alpha\left(x_{5}\right)\right) \\
& +\nearrow\left(\left\{x_{2}, x_{4}, x_{1}\right\}^{v}, \alpha\left(x_{3}\right), \alpha\left(x_{5}\right)\right), \\
r_{5} & =\nearrow\left(\alpha\left(x_{2}\right), \alpha\left(x_{1}\right),\left\{x_{3}, x_{4}, x_{5}\right\}^{h}\right)-\nwarrow\left(\alpha\left(x_{3}\right), \alpha\left(x_{4}\right), \nearrow\left(x_{2}, x_{1}, x_{5}\right)\right)-\nearrow\left(\left\{x_{1}, x_{3}, x_{2}\right\}^{v}, \alpha\left(x_{4}\right), \alpha\left(x_{5}\right)\right) \\
& +\nearrow\left(\left\{x_{1}, x_{4}, x_{2}\right\}^{v}, \alpha\left(x_{3}\right), \alpha\left(x_{5}\right)\right) .
\end{aligned}
$$

From identities (3.36)-(3.38), we obtain immediately $r_{i}=0, \forall 1 \leq i \leq 5$. This implies that Eq.(2.16) holds.

On the other hand, we have

$\left\{\left[x_{1}, x_{2}, x_{3}\right]^{C}, \alpha\left(x_{4}\right), \alpha\left(x_{5}\right)\right\}^{h}-\left\{\alpha\left(x_{1}\right), \alpha\left(x_{2}\right),\left\{x_{3}, x_{4}, x_{5}\right\}^{h}\right\}^{h}-\left\{\alpha\left(x_{2}\right), \alpha\left(x_{3}\right),\left\{x_{1}, x_{4}, x_{5}\right\}^{h}\right\}^{h}-\left\{\alpha\left(x_{3}\right), \alpha\left(x_{1}\right),\left\{x_{2}, x_{4}, x_{5}\right\}^{h}\right\}^{h}$ $=s_{1}+s_{2}+s_{3}+s_{4}+s_{5}$,

where

$$
\begin{gathered}
s_{1}=\nwarrow\left(\left[x_{1}, x_{2}, x_{3}\right]^{C}, \alpha\left(x_{4}\right), \alpha\left(x_{5}\right)\right)-\cup_{1,2,3} \nwarrow\left(\alpha\left(x_{1}\right), \alpha\left(x_{2}\right), \nwarrow\left(x_{3}, x_{4}, x_{5}\right)\right), \\
s_{2}=\nearrow\left(\alpha\left(x_{4}\right),\left[x_{1}, x_{2}, x_{3}\right]^{C}, \alpha\left(x_{5}\right)\right)-\cup_{1,2,3} \nwarrow\left(\alpha\left(x_{1}\right), \alpha\left(x_{2}\right), \nearrow\left(x_{4}, x_{3}, x_{5}\right)\right), \\
s_{3}=\nwarrow\left(\alpha\left(x_{1}\right), \alpha\left(x_{2}\right), \nearrow\left(x_{3}, x_{4}, x_{5}\right)\right)+\nearrow\left(\alpha\left(x_{3}\right), \alpha\left(x_{1}\right),\left\{x_{2}, x_{3}, x_{5}\right\}^{h}\right) \\
\quad-\nearrow\left(\alpha\left(x_{3}\right), \alpha\left(x_{2}\right),\left\{x_{1}, x_{4}, x_{5}\right\}^{h}\right)-\nearrow\left(\left\{x_{1}, x_{2}, x_{3}\right\}^{v}, \alpha\left(x_{4}\right), \alpha\left(x_{5}\right)\right), \\
s_{4}=\nwarrow\left(\alpha\left(x_{2}\right), \alpha\left(x_{3}\right), \nearrow\left(x_{1}, x_{4}, x_{5}\right)\right)+\nearrow\left(\alpha\left(x_{1}\right), \alpha\left(x_{2}\right),\left\{x_{3}, x_{4}, x_{5}\right\}^{h}\right) \\
\quad-\nearrow\left(\alpha\left(x_{1}\right), \alpha\left(x_{3}\right),\left\{x_{2}, x_{4}, x_{5}\right\}^{h}\right)-\nearrow\left(\left\{x_{2}, x_{3}, x_{1}\right\}^{v}, \alpha\left(x_{4}\right), \alpha\left(x_{5}\right)\right), \\
s_{5}=\nwarrow\left(\alpha\left(x_{3}\right), \alpha\left(x_{1}\right), \nearrow\left(x_{2}, x_{4}, x_{5}\right)\right)+\nearrow\left(\alpha\left(x_{2}\right), \alpha\left(x_{3}\right),\left\{x_{1}, x_{4}, x_{5}\right\}^{h}\right) \\
\quad-\nearrow\left(\alpha\left(x_{2}\right), \alpha\left(x_{1}\right),\left\{x_{3}, x_{4}, x_{5}\right\}^{h}\right)-\nearrow\left(\left\{x_{3}, x_{1}, x_{2}\right\}^{v}, \alpha\left(x_{4}\right), \alpha\left(x_{5}\right)\right) .
\end{gathered}
$$

From identities (3.39)-(3.41), we obtain immediately $s_{i}=0, \forall 1 \leq i \leq 5$. This implies that Eq.(2.17) holds.

It is straightforward to verify that:

Corollary 3.3. Let $(A, \nwarrow, \nearrow, \alpha)$ be a 3-Hom-L-dendriform algebra. Then the bracket given by (3.44) together with $\alpha$ define a 3-Hom-Lie algebra structure on A which is called the associated 3-Hom-Lie algebra of $(A, \nwarrow, \nearrow, \alpha)$. 
The following Proposition is obvious.

Proposition 3.4. Let $(A, \nwarrow, \nearrow, \alpha)$ be a 3-Hom-L-dendriform algebra. Define $L_{\nwarrow}, R_{\nearrow}: \otimes^{2} A \rightarrow g l(A)$ by

$$
L_{\nwarrow}(x, y) z=\nwarrow(x, y, z), \quad R_{\nearrow}(x, y) z=\nearrow(z, x, y), \forall x, y, z \in A .
$$

Then $\left(A, L_{\nwarrow}, R_{\nearrow}, \alpha\right)$ is a representation of its associated 3-Hom-pre-Lie algebra $(A,\{\cdot, \cdot, \cdot\}, \alpha)$.

Theorem 3.5. Let $(A,\{\cdot, \cdot, \cdot\}, \alpha)$ be a 3-Hom-pre-Lie algebra and $(V, l, r, \phi)$ be a representation. Suppose that $T: V \rightarrow A$ is an $O$-operator associated to $(V, l, r, \phi)$. Then $(V, \nwarrow, \nearrow, \phi)$ is a 3-Hom-L-dendriform algebra, where

$$
\nwarrow(u, v, w)=l(T u, T v) w, \quad \nearrow(u, v, w)=r(T v, T w) u, \forall u, v, w \in V .
$$

Therefore, there exists an associated 3-Hom-pre-Lie algebra structure on $V$ and $T$ is a homomorphism of 3-Hom-pre-Lie algebras. Moreover, $T(V)=\{T(v) \mid v \in V\}$ is 3-Hom-pre-Lie subalgebra of $(A,\{\cdot, \cdot, \cdot\}, \alpha)$ and there is an induced 3-Hom-L-dendriform algebra structure on $T(V)$ given by

$$
\nwarrow(T u, T v, T w)=T(\nwarrow(u, v, w)), \quad \nearrow(T u, T v, T w)=T(\nearrow(u, v, w)), \forall u, v, w \in V .
$$

Proof. Define $\{\cdot, \cdot, \cdot\}_{V}^{h},\{\cdot, \cdot, \cdot\}_{V}^{v},[\cdot, \cdot, \cdot]_{V}^{C}: \otimes^{3} V \rightarrow V$ by

$$
\begin{aligned}
& \{u, v, w\}_{V}^{h}=\nwarrow(u, v, w)+\nearrow(u, v, w)-\nearrow(v, u, w), \\
& \{u, v, w\}_{V}^{v}=\nwarrow(u, v, w)+\nearrow(w, u, v)-\nearrow(w, v, u), \\
& {[u, v, w]_{V}^{C}=\bigcup_{u, v, w}\{u, v, w\}_{V}^{h}=\cup_{u, v, w}\{u, v, w\}_{V}^{v} .}
\end{aligned}
$$

for any $u, v, w \in V$.

Using identity (2.34), we have

$$
\begin{aligned}
T\{u, v, w\}_{V}^{h} & =T(\nwarrow(u, v, w)+\nearrow(u, v, w)-\nearrow(v, u, w)) \\
& =T(l(T u, T v) w-r(T u, T w) v+r(T v, T w) u)=\{T u, T v, T w\}^{h}
\end{aligned}
$$

and

$$
T[u, v, w]_{V}^{C}=\cup_{u, v, w} T\{u, v, w\}_{V}=\cup_{u, v, w}\{T u, T v, T w\}^{h}=[T u, T v, T w]^{C} .
$$

It is obvious that

$$
\nwarrow(u, v, w)+\nwarrow(v, u, w)=(l(T u, T v)+l(T v, T u)) w=0 .
$$

Furthermore, for any $u_{i} \in V, 1 \leq i \leq 5$, we have

$$
\begin{aligned}
& \nwarrow\left(\phi\left(u_{1}\right), \phi\left(u_{2}\right), \nwarrow\left(u_{3}, u_{4}, u_{5}\right)\right)-\nwarrow\left(\phi\left(u_{3}\right), \phi\left(u_{4}\right), \nwarrow\left(u_{1}, u_{2}, u_{5}\right)\right) \\
& -\nwarrow\left(\left[u_{1}, u_{2}, u_{3}\right]_{V}^{C}, \phi\left(u_{4}\right), \phi\left(u_{5}\right)\right)+\nwarrow\left(\left[u_{1}, u_{2}, u_{4}\right]_{V^{\prime}}^{C} \phi\left(u_{3}\right), \phi\left(u_{5}\right)\right) \\
& =l\left(T\left(\phi\left(u_{1}\right)\right), T\left(\phi\left(u_{2}\right)\right)\right) l\left(T\left(u_{3}\right), T\left(u_{4}\right)\right) u_{5}-l\left(T\left(\phi\left(u_{3}\right)\right), T\left(\phi\left(u_{4}\right)\right)\right) l\left(T\left(u_{1}\right), T\left(u_{2}\right)\right) u_{5} \\
& -l\left(T\left[u_{1}, u_{2}, u_{3}\right]_{V}^{C}, T\left(\phi\left(u_{4}\right)\right)\right) \phi\left(u_{5}\right)+l\left(T\left[u_{1}, u_{2}, u_{4}\right]_{V}^{C}, T\left(\phi\left(u_{3}\right)\right)\right) \phi\left(u_{5}\right) \\
& =l\left(\alpha\left(T\left(u_{1}\right)\right), \alpha\left(T\left(u_{2}\right)\right)\right) l\left(T\left(u_{3}\right), T\left(u_{4}\right)\right) u_{5}-l\left(\alpha\left(T\left(u_{3}\right)\right), \alpha\left(T\left(u_{4}\right)\right)\right) l\left(T\left(u_{1}\right), T\left(u_{2}\right)\right) u_{5} \\
& -l\left(T\left[u_{1}, u_{2}, u_{3}\right]_{V^{\prime}}^{C} \alpha\left(T\left(u_{4}\right)\right)\right) \phi\left(u_{5}\right)+l\left(T\left[u_{1}, u_{2}, u_{4}\right]_{V}^{C}, \alpha\left(T\left(u_{3}\right)\right)\right) \phi\left(u_{5}\right)=0 .
\end{aligned}
$$

This implies that (3.36) holds. Moreover, (3.37) holds. Indeed,

$$
\nearrow\left(\phi\left(u_{5}\right),\left[u_{1}, u_{2}, u_{3}\right]_{V}^{C}, \phi\left(u_{4}\right)\right)-\nearrow\left(\phi\left(u_{5}\right), \phi\left(u_{3}\right),\left\{u_{1}, u_{2}, u_{4}\right\}_{V}^{h}\right)
$$




$$
\begin{aligned}
& -\nwarrow\left(\phi\left(u_{1}\right), \phi\left(u_{2}\right), \nearrow\left(u_{5}, u_{3}, u_{4}\right)\right)+\nearrow\left(\left\{u_{1}, u_{2}, u_{5}\right\}_{V}^{v}, \phi\left(u_{3}\right), \phi\left(u_{4}\right)\right) \\
& =r\left(T\left[u_{1}, u_{2}, u_{3}\right]_{V}^{C}, T\left(\phi\left(u_{4}\right)\right)\right) \phi\left(u_{5}\right)-r\left(T\left(\phi\left(u_{3}\right)\right), T\left\{u_{1}, u_{2}, u_{4}\right\}_{V}^{h}\right) \phi\left(u_{5}\right) \\
& -l\left(T\left(\phi\left(u_{1}\right)\right), T\left(\phi\left(u_{2}\right)\right)\right) r\left(T\left(u_{3}\right), T\left(u_{4}\right)\right) u_{5}+r\left(T\left(\phi\left(u_{3}\right)\right), T\left(\phi\left(u_{4}\right)\right)\right)\left\{u_{1}, u_{2}, u_{5}\right\}_{V}^{v} \\
& =r\left(\left[T u_{1}, T u_{2}, T u_{3}\right]^{C}, \alpha\left(T\left(u_{4}\right)\right)\right) \phi\left(u_{5}\right)-r\left(\alpha\left(T\left(u_{3}\right)\right),\left\{T\left(u_{1}\right), T\left(u_{2}\right), T\left(u_{4}\right)\right\}^{h}\right) \phi\left(u_{5}\right) \\
& -l\left(\alpha\left(T\left(u_{1}\right)\right), \alpha\left(T\left(u_{2}\right)\right)\right) r\left(T\left(u_{3}\right), T\left(u_{4}\right)\right) u_{5}+r\left(\alpha\left(T\left(u_{3}\right)\right), \alpha\left(T\left(u_{4}\right)\right)\right) \mu\left(T\left(u_{1}\right), T\left(u_{2}\right)\right) u_{5}=0 .
\end{aligned}
$$

To prove identity (3.41), we compute as follows

$$
\begin{aligned}
& \nwarrow\left(\phi\left(u_{1}\right), \phi\left(u_{2}\right), \nearrow\left(u_{5}, u_{3}, u_{4}\right)\right)+\nearrow\left(\phi\left(u_{5}\right), \phi\left(u_{1}\right),\left\{u_{2}, u_{3}, u_{4}\right\}_{V}^{h}\right) \\
& \quad-\nearrow\left(\phi\left(u_{5}\right), \phi\left(u_{2}\right),\left\{u_{1}, u_{3}, u_{4}\right\}_{V}^{h}\right)-\nearrow\left(\left\{u_{1}, u_{2}, u_{5}\right\}_{V}^{v}, \phi\left(u_{3}\right), \phi\left(u_{4}\right)\right) \\
& =l\left(\alpha\left(T u_{1}\right), \alpha\left(T u_{2}\right)\right) r\left(T u_{3}, T u_{4}\right) u_{5}+r\left(\alpha\left(T u_{1}\right),\left\{T u_{2}, T u_{3}, T u_{4}\right\}^{h}\right) \phi\left(u_{5}\right) \\
& \quad-r\left(\alpha\left(T u_{2}\right),\left\{T u_{1}, T u_{3}, T u_{4}\right\}^{h}\right) \phi\left(u_{5}\right)-r\left(\alpha\left(T u_{3}\right), \alpha\left(T u_{4}\right)\right) \mu\left(T u_{1}, T u_{2}\right) u_{5}=0 .
\end{aligned}
$$

The other identities can be shown similarly.

Corollary 3.6. Let $(A,\{\cdot, \cdot, \cdot\}, \alpha)$ be a 3-Hom-pre-Lie algebra and $\mathcal{R}: A \rightarrow A$ is a Rota-Baxter operator of weight 0 . Then $(A, \nwarrow, \nearrow, \alpha)$ is a 3-Hom-L-dendriform algebra, where the operations $\nwarrow$ and $\nearrow$ are given by

$$
\nwarrow(x, y, z)=L(\mathcal{R} x, \mathcal{R} y) z=\{\mathcal{R} x, \mathcal{R} y, z\}, \quad \nearrow(x, y, z)=R(\mathcal{R} y, \mathcal{R} z) x=\{x, \mathcal{R} y, \mathcal{R} z\},
$$

for all $x, y, z \in A$.

Corollary 3.7. Let $(A,\{\cdot, \cdot, \cdot\}, \alpha)$ be a 3-Hom-pre-Lie algebra. Then there exists a compatible 3-Hom-Ldendriform algebra if and only if there exists an invertible $O$-operator on $A$.

Proof. Let $T$ be an invertible $O$-operator of $A$ associated to a representation $(V, l, r, \phi)$. Then there exists a 3-Hom-L-dendriform algebra structure on $V$ defined by

$$
\nwarrow(u, v, w)=l(T u, T v) w, \quad \nearrow(u, v, w)=r(T v, T w) u, \forall u, v, w \in V .
$$

In addition there exists a 3-Hom-L-dendriform algebra structure on $T(V)=A$ given by

$$
\nwarrow(T u, T v, T w)=T(l(T u, T v) w), \quad \nearrow(T u, T v, T w)=T(r(T v, T w) u), \forall u, v, w \in V .
$$

If we put $x=T u, y=T v$ and $z=T w$, we get

$$
\nwarrow(x, y, z)=T\left(l(x, y) T^{-1}(z)\right), \quad \nearrow(x, y, z)=T\left(r(y, z) T^{-1}(x)\right), \forall x, y, z \in A .
$$

It is a compatible 3-Hom-L-dendriform algebra structure on $A$. Indeed,

$$
\begin{aligned}
& \nwarrow(x, y, z)+\nearrow(x, y, z)-\nearrow(y, x, z) \\
= & T\left(l(x, y) T^{-1}(z)\right)+T\left(r(y, z) T^{-1}(x)\right)-T\left(r(x, z) T^{-1}(y)\right) \\
= & \left\{T T^{-1}(x), T T^{-1}(y), T T^{-1}(z)\right\}=\{x, y, z\} .
\end{aligned}
$$

Conversely, let $(A,\{\cdot, \cdot, \cdot\}, \alpha)$ be a 3-Hom-pre-Lie algebra and $(A, \nwarrow, \nearrow, \alpha)$ its compatible 3-Hom-Ldendriform algebra. Then the identity map id :A $\rightarrow A$ is an $O$-operator of $(A,\{\cdot, \cdot, \cdot\}, \alpha)$ associated to $(A, L, R, \alpha)$. 
Definition 3.2. Let $(A,\{\cdot, \cdot, \cdot\}, \alpha)$ be a 3-Hom-pre-Lie algebra and $B$ be a skew-symmetric bilinear form on $A$. We say that $B$ is closed if it satisfies for any $x, y, z, w \in A$

$$
B\left(\{\alpha(x), \alpha(y), \alpha(z)\}, \alpha^{2}(w)\right)-B\left(\alpha(z),[x, y, w]^{C}\right)-B(\alpha(y),\{w, x, z\})+B(\alpha(x),\{w, y, z\})=0 .
$$

If in addition $B$ is nondegenerate, then $B$ is called symplectic.

A 3-Hom-pre-Lie algebra $(A,\{\cdot, \cdot, \cdot\}, \alpha)$ equipped with a symplectic form is called a symplectic 3-Hom-pre-Lie algebra and denoted by $(A,\{\cdot, \cdot, \cdot\}, \alpha, B)$.

Proposition 3.8. Let $(A,\{\cdot, \cdot, \cdot\}, \alpha, B)$ be a symplectic 3-Hom-pre-Lie algebra. Then there exists a compatible 3-Hom-L-dendriform algebra structure on A given by

$$
B\left(\nwarrow(\alpha(x), \alpha(y), z), \alpha^{2}(w)\right)=B\left(z,[x, y, w]^{C}\right), \quad B\left(\nearrow(x, \alpha(y), \alpha(z)), \alpha^{2}(w)\right)=-B(x,\{w, y, z\})
$$

for all $x, y, z, w \in A$.

Proof. Define the linear map $T: A^{*} \rightarrow A$ by $\left\langle T^{-1} x, y\right\rangle=B(x, y)$. Since $B$ is $\alpha$-symmetric and using Eq. (3.51), we obtain that $T$ is an invertible $O$-operator on $A$ associated to the coadjoint representation $\left(A^{*}, a d^{\star},-R^{\star},\left(\alpha^{-1}\right)^{*}\right)$. By Corollary 3.7, there exists a compatible 3-Hom-L-dendriform algebra structure given by

$$
\nwarrow(x, y, z)=T\left(a d^{\star}(x, y) T^{-1}(z)\right), \quad \nearrow(x, y, z)=-T\left(R^{\star}(y, z) T^{-1}(x)\right), \forall x, y, z \in A .
$$

Let $x, y, z, w \in A$, then we have

$$
\begin{aligned}
B\left(\nwarrow(\alpha(x), \alpha(y), z), \alpha^{2}(w)\right) & =B\left(T\left(a d^{\star}(\alpha(x), \alpha(y)) T^{-1}(z)\right), \alpha^{2}(w)\right)=\left\langle a d^{\star}(\alpha(x), \alpha(y)) T^{-1}(z), \alpha^{2}(w)\right\rangle \\
& =\left\langle T^{-1}(z),[x, x, w]^{C}\right\rangle=B\left(z,[x, y, w]^{C}\right)
\end{aligned}
$$

and

$$
\begin{aligned}
B\left(\nearrow(x, \alpha(y), \alpha(z)), \alpha^{2}(w)\right) & \left.\left.=-B\left(T\left(R^{\star}(\alpha(y), \alpha(z))\right) T^{-1}(x)\right)\right), \alpha^{2}(w)\right)=-\left\langle R^{\star}(\alpha(y), \alpha(z)) T^{-1}(x), \alpha^{2}(w)\right\rangle \\
& =-\left\langle T^{-1}(x),\{w, y, z\}\right\rangle=-B(x,\{w, y, z\}) .
\end{aligned}
$$

The proof is finished.

Corollary 3.9. Let $(A,\{\cdot, \cdot, \cdot\}, \alpha, B)$ be a symplectic 3 -Hom-pre-Lie algebra and $\left(A,[\cdot, \cdot, \cdot]^{C}, \alpha\right)$ be its associated 3-Hom-Lie algebra. Then there exists a 3-Hom-pre-Lie algebraic structure $\left(A,\{\cdot, \cdot, \cdot\}^{\prime}, \alpha\right)$ on A given by

$$
B\left(\{\alpha(x), \alpha(y), \alpha(z)\}^{\prime}, \alpha^{2}(w)\right)=B\left(\alpha(z),[x, y, w]^{C}\right)-B(\alpha(z),\{w, x, y\})+B(\alpha(z),\{w, y, x\}) .
$$

Lemma 3.10. Let $\left\{\mathcal{R}_{1}, \mathcal{R}_{2}\right\}$ be a pair of of commuting Rota-Baxter operators of weight zero on a 3-Hom-Lie algebra $(A,[\cdot, \cdot, \cdot], \alpha)$. Then $\mathcal{R}_{2}$ is a Rota-Baxter operator of weight zero on the associated 3-Hom-pre-Lie algebra defined by $\{x, y, z\}=\left[\mathcal{R}_{1}(x), \mathcal{R}_{1}(y), z\right]$.

Proof. For any $x, y, z \in A$, we have

$$
\begin{aligned}
& \left\{\mathcal{R}_{2}(x), \mathcal{R}_{2}(y), \mathcal{R}_{2}(z)\right\}=\left[\mathcal{R}_{1} \mathcal{R}_{2}(x), \mathcal{R}_{1} \mathcal{R}_{2}(y), \mathcal{R}_{2}(z)\right]=\left[\mathcal{R}_{2} \mathcal{R}_{1}(x), \mathcal{R}_{2} \mathcal{R}_{1}(y), \mathcal{R}_{2}(z)\right] \\
& =\mathcal{R}_{2}\left(\left[\mathcal{R}_{2} \mathcal{R}_{1}(x), \mathcal{R}_{2} \mathcal{R}_{1}(y), z\right]+\left[\mathcal{R}_{1}(x), \mathcal{R}_{2} \mathcal{R}_{1}(y), \mathcal{R}_{2}(z)\right]+\left[\mathcal{R}_{2} \mathcal{R}_{1}(x), \mathcal{R}_{1}(y), \mathcal{R}_{2}(z)\right]\right) \\
& =\mathcal{R}_{2}\left(\left\{\mathcal{R}_{2}(x), \mathcal{R}_{2}(y), z\right\}+\left\{x, \mathcal{R}_{2}(y), \mathcal{R}_{2}(z)\right\}+\left\{\mathcal{R}_{2}(x), y, \mathcal{R}_{2}(z)\right\}\right) .
\end{aligned}
$$

Hence $\mathcal{R}_{2}$ is a Rota-Baxter operator of weight zero on the 3-Hom-pre-Lie algebra $(A,\{\cdot, \cdot, \cdot\}, \alpha)$. 
Proposition 3.11. Let $\left\{\mathcal{R}_{1}, \mathcal{R}_{2}\right\}$ be a pair of commuting Rota-Baxter operators of weight zero on a 3-HomLie algebra $(A,[\cdot, \cdot, \cdot], \alpha)$. Then $(A, \nwarrow, \nearrow, \alpha)$ is a 3-Hom-L-dendriform algebra, where the two brackets are defined by:

$$
\nwarrow(x, y, z)=\left[\mathcal{R}_{1} \mathcal{R}_{2}(x), \mathcal{R}_{1} \mathcal{R}_{2}(y), z\right], \quad \nearrow(x, y, z)=\left[\mathcal{R}_{1}(x), \mathcal{R}_{1} \mathcal{R}_{2}(y), \mathcal{R}_{2}(z)\right], \forall x, y, z \in A .
$$

Proof. It follows immediately from Lemma 3.10 and Corollary 3.6 ,

Remark 3.12. Let $(A,[\cdot, \cdot], \alpha)$ be a Hom-Lie algebra. Recall that a trace function $\tau: A \rightarrow \mathbb{K}$ is a linear map such that $\tau([x, y])=0$ and $\tau \alpha=\tau, \forall x, y \in A$. When $\tau$ is a trace function, it is well known [7] that $\left(A,[\cdot, \cdot, \cdot]_{\tau}, \alpha\right)$ is a 3-Hom-Lie algebra, where

$$
[x, y, z]_{\tau}:=\cup_{x, y, z \in A} \tau(x)[y, z], \forall x, y, z \in A .
$$

Now, let $\left\{\mathcal{R}_{1}, \mathcal{R}_{2}\right\}$ be a pair of commuting Rota-Baxter operators of weight zero on the 3-Hom-Lie algebra $\left(A,[\cdot, \cdot, \cdot]_{\tau}, \alpha\right)$. Then we can construct a 3-Hom-L-dendriform structure on $A$, given by

$$
\begin{aligned}
& \nwarrow(x, y, z)=\tau\left(\mathcal{R}_{1} \mathcal{R}_{2}(x)\right)\left[\mathcal{R}_{1} \mathcal{R}_{2}(y), z\right]+\tau\left(\mathcal{R}_{1} \mathcal{R}_{2}(y)\right)\left[z, \mathcal{R}_{1} \mathcal{R}_{2}(x)\right]++\tau(z)\left[\mathcal{R}_{1} \mathcal{R}_{2}(x), \mathcal{R}_{1} \mathcal{R}_{2}(y)\right], \\
& \nearrow(x, y, z)=\tau\left(\mathcal{R}_{1}(x)\right)\left[\mathcal{R}_{1} \mathcal{R}_{2}(y), \mathcal{R}_{2}(z)\right]+\tau\left(\mathcal{R}_{1} \mathcal{R}_{2}(y)\right)\left[\mathcal{R}_{2}(z), \mathcal{R}_{1}(x)\right]+\tau\left(\mathcal{R}_{2}(z)\right)\left[\mathcal{R}_{1}(x), \mathcal{R}_{1} \mathcal{R}_{2}(y)\right],
\end{aligned}
$$

for any $x, y, z \in A$.

\section{Nijenhuis Operators on 3-Hom-L-dendriform algebras}

In this section, we study the second order deformation of 3-Hom-L-dendriform algebras and introduce the notion of Nijenhuis operator on 3-Hom-L-dendriform algebras, which could generate a trivial deformation. Moreover, we give some properties and results of Nijenhuis operators.

\subsection{Second-order deformation of 3-Hom-L-dendriform algebras}

Let $(A, \nwarrow, \nearrow, \alpha)$ be a 3-Hom-L-dendriform algebra and $\omega_{\nwarrow}^{i}, \omega^{i}, A \times A \times A \longrightarrow A, i=1,2$ be a multilinear maps. Consider a $\lambda$-parameterized family of 3-linear operations:

$$
\begin{aligned}
& \nwarrow_{\lambda}\left(x_{1}, x_{2}, x_{3}\right)=\nwarrow\left(x_{1}, x_{2}, x_{3}\right)+\lambda \omega_{\nwarrow}^{1}\left(x_{1}, x_{2}, x_{3}\right)+\lambda^{2} \omega_{\nwarrow}^{2}\left(x_{1}, x_{2}, x_{3}\right)+\ldots, \\
& \nearrow_{\lambda}\left(x_{1}, x_{2}, x_{3}\right)=\nearrow\left(x_{1}, x_{2}, x_{3}\right)+\lambda \omega_{\nearrow}^{1}\left(x_{1}, x_{2}, x_{3}\right)+\lambda^{2} \omega_{\nearrow}^{2}\left(x_{1}, x_{2}, x_{3}\right)+\ldots,
\end{aligned}
$$

where $\lambda \in \mathbb{K}$. If all $\left(\nwarrow_{\lambda}, \nearrow_{\lambda}, \alpha\right)$ are 3-Hom-L-dendriform algebra structures, we say that $\omega_{\nwarrow}^{i}, \omega_{\nwarrow}^{i}$ generates a $\lambda$-parameter deformation of the 3-Hom-L-dendriform algebra $(A, \nwarrow, \nearrow, \alpha)$.

Remark 4.1. 1. If $\lambda^{2}=0$, the deformation is called infinitesimal.

2. If $\lambda^{n}=0$, the deformation is said to be of order $n-1$.

Definition 4.1. A deformation is said to be trivial if there exists an linear map $N: A \longrightarrow A$ such that for all $\lambda, T_{\lambda}=i d+\lambda N$ satisfies

$$
\begin{aligned}
T_{\lambda} \circ \alpha & =\alpha \circ T_{\lambda}, \\
T_{\lambda} \nwarrow_{\lambda}\left(x_{1}, x_{2}, x_{3}\right) & =\nwarrow\left(T_{\lambda} x_{1}, T_{\lambda} x_{2}, T_{\lambda} x_{3}\right), \\
T_{\lambda} \nearrow_{\lambda}\left(x_{1}, x_{2}, x_{3}\right) & =\nearrow\left(T_{\lambda} x_{1}, T_{\lambda} x_{2}, T_{\lambda} x_{3}\right), \forall x_{1}, x_{2}, x_{3} \in A .
\end{aligned}
$$


Eq. (4.57) is equals to $N \circ \alpha=\alpha \circ N$. The left hand side of Eq. (4.58) is equivalent to

$$
\begin{aligned}
& \nwarrow\left(x_{1}, x_{2}, x_{3}\right)+\lambda\left(\omega_{\nwarrow}^{1}\left(x_{1}, x_{2}, x_{3}\right)+N \nwarrow\left(x_{1}, x_{2}, x_{3}\right)\right) \\
& +\lambda^{2}\left(\omega_{\nwarrow}^{2}\left(x_{1}, x_{2}, x_{3}\right)+N \omega_{\nwarrow}^{1}\left(x_{1}, x_{2}, x_{3}\right)\right)+\lambda^{3} N \omega_{\nwarrow}^{2}\left(x_{1}, x_{2}, x_{3}\right)+\ldots
\end{aligned}
$$

The right hand side of Eq. (4.58) can be written as

$$
\begin{aligned}
& \nwarrow\left(x_{1}, x_{2}, x_{3}\right)+\lambda\left(\nwarrow\left(N x_{1}, x_{2}, x_{3}+\nwarrow\left(x_{1}, N x_{2}, x_{3}\right)+\nwarrow\left(x_{1}, x_{2}, N x_{3}\right)\right)\right. \\
& +\lambda^{2}\left(\nwarrow\left(N x_{1}, N x_{2}, x_{3}\right)+\nwarrow\left(N x_{1}, x_{2}, N x_{3}\right)+\nwarrow\left(x_{1}, N x_{2}, N x_{3}\right)\right)+\lambda^{3} \nwarrow\left(N x_{1}, N x_{2}, N x_{3}\right) .
\end{aligned}
$$

Therefore, by Eq. (4.58), we have

$$
\begin{aligned}
& \omega_{\nwarrow}^{1}\left(x_{1}, x_{2}, x_{3}\right)+N \nwarrow\left(x_{1}, x_{2}, x_{3}\right)=\nwarrow\left(N x_{1}, x_{2}, x_{3}\right)+\nwarrow\left(x_{1}, N x_{2}, x_{3}\right)+\nwarrow\left(x_{1}, x_{2}, N x_{3}\right), \\
& \omega_{\nwarrow}^{2}\left(x_{1}, x_{2}, x_{3}\right)+N \omega_{\nwarrow}^{1}\left(x_{1}, x_{2}, x_{3}\right)=\nwarrow\left(N x_{1}, N x_{2}, x_{3}\right)+\nwarrow\left(N x_{1}, x_{2}, N x_{3}\right)+\nwarrow\left(x_{1}, N x_{2}, N x_{3}\right), \\
& N \omega_{\nwarrow}^{2}\left(x_{1}, x_{2}, x_{3}\right)=\nwarrow\left(N x_{1}, N x_{2}, N x_{3}\right) .
\end{aligned}
$$

Similarly, using (4.59) we obtain

$$
\begin{aligned}
& \omega_{\nearrow}^{1}\left(x_{1}, x_{2}, x_{3}\right)+N \nearrow\left(x_{1}, x_{2}, x_{3}\right)=\nearrow\left(N x_{1}, x_{2}, x_{3}\right)+\nearrow\left(x_{1}, N x_{2}, x_{3}\right)+\nearrow\left(x_{1}, x_{2}, N x_{3}\right), \\
& \omega_{\nearrow}^{2}\left(x_{1}, x_{2}, x_{3}\right)+N \omega_{\nearrow}^{1}\left(x_{1}, x_{2}, x_{3}\right)=\nearrow\left(N x_{1}, N x_{2}, x_{3}\right)+\nearrow\left(N x_{1}, x_{2}, N x_{3}\right)+\nearrow\left(x_{1}, N x_{2}, N x_{3}\right), \\
& N \omega_{\nearrow}^{2}\left(x_{1}, x_{2}, x_{3}\right)=\nearrow\left(N x_{1}, N x_{2}, N x_{3}\right) .
\end{aligned}
$$

Let $(A, \nwarrow, \nearrow, \alpha)$ be a 3-Hom-L-dendriform algebra, and $N: A \longrightarrow A$ a linear map. Define a ternary operations $\nwarrow_{N^{\prime}}^{1} \nearrow_{N}^{1}: \otimes^{3} A \longrightarrow A$ by

$$
\begin{aligned}
& \nwarrow_{N}^{1}\left(x_{1}, x_{2}, x_{3}\right)=\nwarrow\left(N x_{1}, x_{2}, x_{3}\right)+\nwarrow\left(x_{1}, N x_{2}, x_{3}\right)+\nwarrow\left(x_{1}, x_{2}, N x_{3}\right)-N \nwarrow\left(x_{1}, x_{2}, x_{3}\right), \\
& \nearrow_{N}^{1}\left(x_{1}, x_{2}, x_{3}\right)=\nearrow\left(N x_{1}, x_{2}, x_{3}\right)+\nearrow\left(x_{1}, N x_{2}, x_{3}\right)+\nearrow\left(x_{1}, x_{2}, N x_{3}\right)-N \nearrow\left(x_{1}, x_{2}, x_{3}\right) .
\end{aligned}
$$

Then we define ternary operations $\nwarrow_{N^{\prime}}^{2} \nearrow_{N}^{2}: \otimes^{3} A \longrightarrow A$, via induction by

$$
\begin{aligned}
& \nwarrow_{N}^{2}\left(x_{1}, x_{2}, x_{3}\right)=\nwarrow\left(N x_{1}, N x_{2}, x_{3}\right)+\nwarrow\left(N x_{1}, x_{2}, N x_{3}\right)+\nwarrow\left(x_{1}, N x_{2}, N x_{3}\right)-N \nwarrow_{N}^{1}\left(x_{1}, x_{2}, x_{3}\right) . \\
& \nearrow_{N}^{2}\left(x_{1}, x_{2}, x_{3}\right)=\nearrow\left(N x_{1}, N x_{2}, x_{3}\right)+\nearrow\left(N x_{1}, x_{2}, N x_{3}\right)+\nearrow\left(x_{1}, N x_{2}, N x_{3}\right)-N \nearrow_{N}^{1}\left(x_{1}, x_{2}, x_{3}\right) .
\end{aligned}
$$

Definition 4.2. Let $(A, \nwarrow, \nearrow, \alpha)$ be a 3-Hom-L-dendriform algebra. A linear map $N: A \rightarrow A$ is called a Nijenhuis operator if

$$
\begin{aligned}
\alpha \circ N & =N \circ \alpha, \\
\nwarrow\left(N\left(x_{1}\right), N\left(x_{2}\right), N\left(x_{3}\right)\right) & =\sum_{I \subseteq[3]^{*}}(-1)^{|I|-1} N^{|I|} \nwarrow\left(\widetilde{N}\left(x_{1}\right), \widetilde{N}\left(x_{2}\right), \widetilde{N}\left(x_{3}\right)\right), \\
\nearrow\left(N\left(x_{1}\right), N\left(x_{2}\right), N\left(x_{3}\right)\right) & =\sum_{\emptyset \neq I \subseteq[3]^{*}}(-1)^{|I|-1} N^{|I|} \nearrow\left(\widetilde{N}\left(x_{1}\right), \widetilde{N}\left(x_{2}\right), \widetilde{N}\left(x_{3}\right)\right),
\end{aligned}
$$

$\forall x_{1}, x_{2}, x_{3} \in A$, where $\widetilde{N}\left(x_{i}\right)= \begin{cases}x_{i} & i \in I ; \\ N\left(x_{i}\right) & i \notin I .\end{cases}$

The Eqs. (4.65) and (4.66) can be written

$$
\begin{aligned}
\nwarrow(N x, N y, N z)= & N(\nwarrow(N x, N y, z)+\nwarrow(N x, y, N z)+\nwarrow(x, N y, N z) \\
& \left.-N(\nwarrow(N x, y, z)+\nwarrow(x, N y, z)+\nwarrow(x, y, N z))+N^{2} \nwarrow(x, y, z)\right), \\
\nearrow(N(x), N(y), N(z))= & N(\nearrow(N x, N y, z)+\nearrow(N x, y, N z)+\nearrow(x, N y, N z) \\
& \left.-N(\nearrow(N x, y, z)+\nearrow(x, N y, z)+\nearrow(x, y, N z))+N^{2} \nearrow(x, y, z)\right) .
\end{aligned}
$$




\subsection{Some properties of Nijenhuis operators}

Proposition 4.2. Let $N$ be a Nijenhuis operator on a 3-Hom-L-dendriform algebra $(A, \nwarrow, \nearrow, \alpha)$. Then $\left(A, \nwarrow_{N^{\prime}}, \nearrow_{N^{\prime}}^{1} \alpha\right)$ is a 3-Hom-L-dendriform algebra and $N$ is a morphism of 3-Hom-L-dendriform algebra from $\left(A, \nwarrow_{N^{\prime}}^{1} \nearrow_{N}^{1}, \alpha\right)$ to $(A, \nwarrow, \nearrow, \alpha)$, where the two operations $\nwarrow_{N^{\prime}}^{1} \nearrow_{N}^{1}$ are defined above by equations (4.60) and (4.61).

Proof. Straightforward.

Proposition 4.3. Let $N$ be a Nijenhuis operator on a 3-Hom-L-dendriform algebras $(A, \nwarrow, \nearrow, \alpha)$. Then $N$ is a Nijenhuis operator on their sub-adjacent 3-Hom-pre-Lie algebras $\left(A,\{\cdot, \cdot, \cdot\}^{h}, \alpha\right),\left(A,\{\cdot, \cdot, \cdot\}^{v}, \alpha\right)$ and its sub-adjacent 3 -Hom-Lie algebra $\left(A,[\cdot, \cdot, \cdot]^{C}, \alpha\right)$.

Proof. Let $x, y, z \in A$, then we have

$$
\begin{aligned}
\{N x, N y, N z\}^{h}= & \nwarrow(N x, N y, N z)+\nearrow(N x, N y, N z)-\nearrow(N y, N x, N z) \\
= & N(\nwarrow(N x, N y, z)+\nwarrow(N x, y, N z)+\nwarrow(x, N y, N z) \\
& \left.-N(\nwarrow(N x, y, z)+\nwarrow(x, N y, z)+\nwarrow(x, y, N z))+N^{2} \nwarrow(x, y, z)\right) \\
& +N(\nearrow(N x, N y, z)+\nearrow(N x, y, N z)+\nearrow(x, N y, N z) \\
& \left.-N(\nearrow(N x, y, z)+\nearrow(x, N y, z)+\nearrow(x, y, N z))+N^{2} \nearrow(x, y, z)\right) \\
& -N(\nearrow(N y, N x, z)-\nearrow(N y, x, N z)-\nearrow(y, N x, N z) \\
& \left.\left.+N(\nearrow(N y, x, z)+\nearrow(y, N x, z)+\nearrow(y, x, N z))-N^{2} \nearrow(y, x, z)\right)\right) \\
= & N\left(\{N x, N y, z\}^{h}+\{N x, y, N z\}^{h}+\{x, N y, N z\}^{h}\right. \\
& \left.-N\left(\{N x, y, z\}^{h}+\{x, N y, z\}^{h}+\{x, y, N z\}^{h}\right)+N^{2}\{x, y, z\}^{h}\right) .
\end{aligned}
$$

Therefor $N$ is a Nijenhuis operator on sub-adjacent 3-Hom-pre-Lie algebras $\left(A,\{\cdot, \cdot, \cdot\}^{h}, \alpha\right)$. Similarly, we can found that $N$ is a Nijenhuis operator on 3-Hom-pre-Lie algebras $\left(A,\{\cdot, \cdot, \cdot\}^{v}, \alpha\right)$ and on the sub-adjacent 3 -Hom-Lie algebra $\left(A,[\cdot, \cdot, \cdot]^{C}, \alpha\right)$.

Proposition 4.4. Let $N$ be a Nijenhuis operator on a 3-Hom-pre-Lie algebra $(A,\{\cdot, \cdot, \cdot\}, \alpha)$ and $R: A \rightarrow A$ be a Rota-Baxter operator such that $N \circ R=R \circ N$. Then $N$ is a Nijenhuis operator on 3-Hom-L-dendriform algebras $(A, \nwarrow, \nearrow, \alpha)$ defined in Corollary 3.6 .

Proof. It can be checked immediately. So we omit details.

It obvious to show that:

Proposition 4.5. Let $(A, \nwarrow, \nearrow, \alpha)$ be a 3-Hom-L-dendriform algebra. If an endomorphism $N$ is a derivation, then $N$ is a Nijenhuis operator if and only if $N$ is a Rota-Baxter operator of weight 0 on $A$.

\subsection{Product and complex structures on 3-Hom-L-dendriform algebras}

Throughout this section, we work over the field $\mathbb{R}$ the complex filed $\mathbb{C}$ and all the vector spaces are fined dimension. We introduce the notion of a product and complex structure on a 3-Hom-Ldendriform algebra using the Nijenhuis condition as the integrability condition.

Definition 4.3. Let $(A, \nwarrow, \nearrow, \alpha)$ be a 3-Hom-L-dendriform algebra. An almost product structure on the 3-Hom-L-dendriform algebra $(A, \nwarrow, \nearrow, \alpha)$ is a linear endomorphism $E: A \rightarrow A$ satisfying $E^{2}=i d_{A}$. An almost product structure is called a product structure if it is a Nijenhuis operator. 
Remark 4.6. One can understand a product structure on the 3-Hom-L-dendriform algebra $(A, \nwarrow, \nearrow, \alpha)$ as an linear map $E: A \rightarrow A$ satisfying

$$
\begin{aligned}
E^{2}=i d_{A}, \quad & E \alpha=\alpha E, \\
E \nwarrow(x, y, z)= & \nwarrow(E x, E y, E z)+\nwarrow(E x, y, z)+\nwarrow(x, E y, z)+\nwarrow(x, y, E z) \\
& -E \nwarrow(E x, E y, z)-E \nwarrow(x, E y, E z)-E \nwarrow(E x, y, E z), \\
E \nearrow(x, y, z)= & \nearrow(E x, E y, E z)+\nearrow(E x, y, z)+\nearrow(x, E y, z)+\nearrow(x, y, E z) \\
& -E \nearrow(E x, E y, z)-E \nearrow(x, E y, E z)-E \nearrow(E x, y, E z) .
\end{aligned}
$$

Theorem 4.7. Let $(A, \nwarrow, \nearrow, \alpha)$ be a 3-Hom-L-dendriform algebra. Then $(A, \nwarrow, \nearrow, \alpha)$ has a product structure if and only if $A$ admits a decomposition:

$$
A=A_{+} \oplus A_{-},
$$

where $A_{+}$and $A_{-}$are subalgebras of $A$.

Proof. Let $E$ be a product structure on $A$. By $E^{2}=i d_{A}$, we have $A=A_{+} \oplus A_{-}$, where $A_{+}$and $A_{-}$are the eigenspaces of $A$ associated to the eigenvalues \pm 1 . For all $x \in A_{+}$, we have $\alpha(x) \in A_{+}$, in fact

$$
E \alpha(x)=\alpha E(x)=\alpha(x)
$$

Now, let $x_{1}, x_{2}, x_{3} \in A_{+}$, we have

$$
\begin{aligned}
E \nwarrow\left(x_{1}, x_{2}, x_{3}\right)= & \nwarrow\left(E x_{1}, E x_{2}, E x_{3}\right)+\nwarrow\left(E x_{1}, x_{2}, x_{3}\right)+\nwarrow\left(x_{1}, E x_{2}, x_{3}\right)+\nwarrow\left(x_{1}, x_{2}, E x_{3}\right) \\
& -E \nwarrow\left(E x_{1}, E x_{2}, x_{3}\right)-E \nwarrow\left(x_{1}, E x_{2}, E x_{3}\right)-E \nwarrow\left(E x_{1}, x_{2}, E x_{3}\right) \\
= & 4 \nwarrow\left(x_{1}, x_{2}, x_{3}\right)-3 E \nwarrow\left(x_{1}, x_{2}, x_{3}\right) .
\end{aligned}
$$

Thus, we have $\nwarrow\left(x_{1}, x_{2}, x_{3}\right) \in A_{+}$. We can also show that $\nearrow\left(x_{1}, x_{2}, x_{3}\right) \in A_{+}$, which implies that $A_{+}$is a subalgebra. Similarly, we can show that $A_{-}$is a subalgebra.

Conversely, we define a linear endomorphism $E: A \rightarrow A$ by

$$
E(x+a)=x-a, \quad \forall x \in A_{+}, a \in A_{-} .
$$

Obviously we have $E^{2}=i d_{A}$ and $E \alpha=\alpha E$.

Since $A_{+}$is a subalgebra of $A$, for all $x_{1}, x_{2}, x_{3} \in A_{+}$, we have

$$
\begin{aligned}
& \nwarrow\left(E x_{1}, E x_{2}, E x_{3}\right)+\nwarrow\left(E x_{1}, x_{2}, x_{3}\right)+\nwarrow\left(x_{1}, E x_{2}, x_{3}\right)+\nwarrow\left(x_{1}, x_{2}, E x_{3}\right) \\
& -E \nwarrow\left(E x_{1}, E x_{2}, x_{3}\right)-E \nwarrow\left(x_{1}, E x_{2}, E x_{3}\right)-E \nwarrow\left(E x_{1}, x_{2}, E x_{3}\right) \\
= & 4 \nwarrow\left(x_{1}, x_{2}, x_{3}\right)-3 E \nwarrow\left(x_{1}, x_{2}, x_{3}\right)=\nwarrow\left(x_{1}, x_{2}, x_{3}\right) \\
= & E \nwarrow\left(x_{1}, x_{2}, x_{3}\right),
\end{aligned}
$$

which implies that (4.67) holds for all $x_{1}, x_{2}, x_{3} \in A_{+}$and by the same computation, we can prove the identity (4.68). Similarly, we can show that (4.67)-(4.68) holds for all $x, y, z \in A$. Therefore, $E$ is a product structure on $A$.

Proposition 4.8. Let E be an almost product structure on a 3-Hom-L-dendriform algebra $(A, \nwarrow, \nearrow, \alpha)$ commuting wilth $\alpha$. 
1. If E satisfies

$$
\begin{aligned}
& E \nwarrow(x, y, z)=\nwarrow(E x, y, z)=\nwarrow(x, y, E z), \\
& E \nearrow(x, y, z)=\nearrow(E x, y, z)=\nearrow(x, E y, z)=\nearrow(x, y, E z), \forall x, y, z \in A .
\end{aligned}
$$

Then $E$ is a product structure on A called a strict product structure.

2. If E satisfies

$$
\begin{aligned}
& \nwarrow(x, y, z)=-\nwarrow(x, E y, E z)-\nwarrow(E x, y, E z)-\nwarrow(E x, E y, z), \\
& \nearrow(x, y, z)=-\nearrow(x, E y, E z)-\nearrow(E x, y, E z)-\nearrow(E x, E y, z), \forall x, y, z \in A .
\end{aligned}
$$

Then $E$ is a product structure on A called an abilian product structure.

3. If E satisfies

$$
\begin{aligned}
& \nwarrow(x, y, z)=E(\nwarrow(x, y, E z)+\nwarrow(E x, y, z)+\nwarrow(x, E y, z)), \\
& \nearrow(x, y, z)=E(\nearrow(x, y, E z)+\nearrow(E x, y, z)+\nearrow(x, E y, z)), \forall x, y, z \in A .
\end{aligned}
$$

Then $E$ is a product structure on A called an strong product structure.

4. If E satisfies

$$
E \nwarrow(x, y, z)=\nwarrow(E x, E y, E z), E \nearrow(x, y, z)=\nearrow(E x, E y, E z), \forall x, y, z \in A .
$$

Then $E$ is a product structure on A called a perfect product structure.

Proof. 1. By (4.76) and $E^{2}=i d_{A}$, we have

$$
\begin{aligned}
& \nwarrow(E x, E y, E z)+\nwarrow(E x, y, z)+\nwarrow(x, E y, z)+\nwarrow(x, y, E z) \\
& -E \nwarrow(E x, E y, z)-E \nwarrow(x, E y, E z)-E \nwarrow(E x, y, E z) \\
= & \nwarrow(E x, E y, E z)+E \nwarrow(x, y, z)+\nwarrow(x, E y, z)+\nwarrow(x, y, E z) \\
& -\nwarrow\left(E^{2} x, E y, z\right)-\nwarrow(E x, E y, E z)-\nwarrow\left(E^{2} x, y, E z\right) \\
= & E \nwarrow(x, y, z) .
\end{aligned}
$$

Similarly, we can prove the identity (4.68). Thus, $E$ is a product structure on $A$.

2. By (4.72) and $E^{2}=i d_{A}$, we have

$$
\begin{aligned}
& \nwarrow(E x, E y, E z)+\nwarrow(E x, y, z)+\nwarrow(x, E y, z)+\nwarrow(x, y, E z) \\
& -E \nwarrow(E x, E y, z)-E \nwarrow(x, E y, E z)-E \nwarrow(E x, y, E z) \\
= & -\nwarrow\left(E x, E^{2} y, E^{2} z\right)-\nwarrow\left(E^{2} x, E y, E^{2} z\right)-\nwarrow\left(E^{2} x, E^{2} y, E z\right) \\
& +\nwarrow(E x, y, z)+\nwarrow(x, E y, z)+\nwarrow(x, y, E z)+E \nwarrow(x, y, z) \\
= & E \nwarrow(x, y, z) .
\end{aligned}
$$

Thus, $E$ is a product structure on $A$.

Items 3. and 4. can be proved similarly.

Remark 4.9. A strict product structure on a 3-Hom-L-dendriform algebra is a perfect product structure. 
Definition 4.4. Let $(A, \nwarrow, \nearrow, \alpha)$ be a 3-Hom-L-dendriform algebra. An almost complex structure on the 3-Hom-L-dendriform algebra $(A, \nwarrow, \nearrow, \alpha)$ is a linear endomorphism $J: A \rightarrow A$ satisfying $J^{2}=-i d_{A}$. An almost structure is called a complex structure if it is a Nijenhuis operator.

Remark 4.10. One can understand a complex structure on the 3-Hom-L-dendriform algebra $(A, \nwarrow, \nearrow, \alpha)$ as an linear map $J: A \rightarrow$ A satisfying

$$
\begin{aligned}
J^{2}=-i d_{A}, \quad & J \alpha=\alpha J, \\
J \nwarrow(x, y, z)= & -\nwarrow(J x, J y, J z)+\nwarrow(J x, y, z)+\nwarrow(x, J y, z)+\nwarrow(x, y, J z) \\
& +J \nwarrow(J x, J y, z)+J \nwarrow(x, J y, J z)+J \nwarrow(J x, y, J z), \\
J \nearrow(x, y, z)= & -\nearrow(J x, J y, J z)+\nearrow(J x, y, z)+\nearrow(x, J y, z)+\nearrow(x, y, J z) \\
& +J \nearrow(J x, J y, z)+J \nearrow(x, J y, J z)+J \nearrow(J x, y, J z) .
\end{aligned}
$$

Remark 4.11. One can also use definition 4.4 to define the notion of a complex structure on a complex 3-Hom-L-dendriform algebra, considering $J$ to be $\mathbb{C}$-linear. However, this is not very interesting since for a complex 3-Hom-L-dendriform algebra, there is a one-to-one correspondence between such $\mathbb{C}$-linear complex structures and product structures.

Consider $A_{\mathbb{C}}=A \otimes_{\mathbb{R}} \mathbb{C} \cong\{x+i y \mid x, y \in A\}$, the complexification of the real 3-Hom-L-dendriform algebra $A$. We will denote it by $\left(A_{\mathbb{C}}, \nwarrow_{A_{\mathbb{C}}}, \nearrow_{A_{\mathbb{C}}}, \alpha_{A_{\mathbb{C}}}\right)$. We have an equivalent description of the integrability condition given in Remark 4.10. For any $z=x+i y \in A_{\mathbb{C}}$ we denote its conjugate by $\bar{z}=x-i y, x, y \in A$. Then the conjugation in $A_{\mathbb{C}}$ is a complex antilinear, involutive automorphism of the complex vector space $A_{\mathbb{C}}$.

Theorem 4.12. Let $(A, \nwarrow, \nearrow, \alpha)$ be a real 3-Hom-L-dendriform algebra. Then $(A, \nwarrow, \nearrow, \alpha)$ has a complex product structure if and only if $A_{\mathbb{C}}$ admits a decomposition:

$$
A_{\mathbb{C}}=\mathfrak{q} \oplus \overline{\mathfrak{q}},
$$

where $\mathfrak{q}$ is a complex subalgebras of $A_{\mathbb{C}}$.

Proof. We extend the complex structure $J \mathbb{C}$-linearly, which is denoted by $J_{\mathbb{C}}$, i.e. $J_{\mathbb{C}}: A_{\mathbb{C}} \longrightarrow A_{\mathbb{C}}$ is defined as

$$
J_{\mathbb{C}}(x+i y)=J x+i J y, \quad \forall x, y \in A .
$$

Then $J_{\mathbb{C}}$ is a $\mathbb{C}$-linear endomorphism on $A_{\mathbb{C}}$ satisfying $J_{\mathbb{C}}^{2}=-i d_{A_{\mathbb{C}}}, J_{\mathbb{C}} \alpha_{\mathbb{C}}=\alpha_{\mathbb{C}} J_{\mathbb{C}}$ and the integrability conditions (4.77) and (4.78) on $A_{\mathbb{C}}$. Denote by $A_{ \pm i}$ the corresponding eigenspaces of $A_{\mathbb{C}}$ associated to the eigenvalues $\pm i$ and there holds:

$$
A_{\mathbb{C}}=A_{i} \oplus A_{-i} .
$$

It is straightforward to see that $A_{i}=\{x-i J x \mid x \in A\}$ and $A_{-i}=\{x+i J x \mid x \in A\}$. Therefore, we have $A_{-i}=\overline{A_{i}}$. It is easy to check that $\alpha_{\mathbb{C}}\left(A_{i}\right) \subset A_{i}$. For all $X, Y, Z \in A_{i}$, we have

$$
\begin{aligned}
J_{\mathbb{C}} \nwarrow_{A_{\mathbb{C}}}(X, Y, Z)= & -\nwarrow_{A_{\mathbb{C}}}\left(J_{\mathbb{C}} X, J_{\mathbb{C}} Y, J_{\mathbb{C}} Z\right)+\nwarrow_{A_{\mathbb{C}}}\left(J_{\mathbb{C}} X, Y, Z\right)+\nwarrow_{A_{\mathbb{C}}}\left(X, J_{\mathbb{C}} Y, Z\right)+\nwarrow_{A_{\mathbb{C}}}\left(X, Y, J_{\mathbb{C}} Z\right) \\
& +J_{\mathbb{C}} \nwarrow_{A_{\mathbb{C}}}\left(J_{\mathbb{C}} X, J_{\mathbb{C}} Y, Z\right)+J_{\mathbb{C}} \nwarrow_{A_{\mathbb{C}}}\left(X, J_{\mathbb{C}} Y, J_{\mathbb{C}} Z\right)+J_{\mathbb{C}} \nwarrow_{A_{\mathbb{C}}}\left(J_{\mathbb{C}} X, Y, J_{\mathbb{C}} Z\right) \\
= & 4 i \nwarrow_{A_{\mathbb{C}}}(X, Y, Z)-3 J_{\mathbb{C}} \nwarrow_{A_{\mathbb{C}}}(X, Y, Z) .
\end{aligned}
$$

Thus, we have $\nwarrow_{A_{\mathbb{C}}}(X, Y, Z) \in A_{i}$. Similarly, we can show that $\nearrow_{A_{\mathbb{C}}}(X, Y, Z) \in A_{i}$, which implies that $A_{i}$ is a subalgebra of $A_{\mathbb{C}}$. 
Conversely, we define a complex linear endomorphism $J_{\mathbb{C}}: A_{\mathbb{C}} \rightarrow A_{\mathbb{C}}$ by

$$
J_{\mathbb{C}}(X+\bar{Y})=i X-i \bar{Y}, \quad \forall X, Y \in \mathfrak{q} .
$$

Since the conjugation is a $\mathbb{C}$-antilinear, involutive automorphism of $A_{\mathbb{C}}$, we have

$$
J_{\mathbb{C}}^{2}(X+\bar{Y})=J_{\mathbb{C}}(i X-i \bar{Y})=J_{\mathbb{C}}(i X+\bar{i} \bar{Y})=i(i X)-i \bar{i} \bar{Y}=-X-\bar{Y}
$$

then $J_{\mathbb{C}}^{2}=-i d$. Moreover, for any $X, Y \in \mathfrak{q}$, we have

$$
\begin{aligned}
J_{\mathbb{C}} \alpha_{\mathbb{C}}(X+\bar{Y}) & =J_{\mathbb{C}}\left(\alpha_{\mathbb{C}}(X)+\alpha_{\mathbb{C}}(\bar{Y})\right)=J_{\mathbb{C}}\left(\alpha_{\mathbb{C}}(X)+\overline{\alpha_{\mathbb{C}}(Y)}\right) \\
& =i \alpha_{\mathbb{C}}(X)-\overline{i \alpha_{\mathbb{C}}(Y)}=\alpha_{\mathbb{C}}(i X)-\alpha_{\mathbb{C}}(i \bar{Y})=\alpha_{\mathbb{C}} J_{\mathbb{C}}(X+\bar{Y}) .
\end{aligned}
$$

Since $\mathfrak{q}$ is a subalgebra of $A_{\mathbb{C}}$ then, for all $X, Y, Z \in \mathfrak{q}$, we have

$$
\begin{aligned}
& -\nwarrow_{A_{\mathbb{C}}}\left(J_{\mathbb{C}} X, J_{\mathbb{C}} Y, J_{\mathbb{C}} Z\right)+\nwarrow_{A_{\mathbb{C}}}\left(J_{\mathbb{C}} X, Y, Z\right)+\nwarrow_{A_{\mathbb{C}}}\left(X, J_{\mathbb{C}} Y, Z\right)+\nwarrow_{A_{\mathbb{C}}}\left(X, Y, J_{\mathbb{C}} Z\right) \\
& +J_{\mathbb{C}} \nwarrow_{A_{\mathbb{C}}}\left(J_{\mathbb{C}} X, J_{\mathbb{C}} Y, Z\right)+J_{\mathbb{C}} \nwarrow_{A_{\mathbb{C}}}\left(X, J_{\mathbb{C}} Y, J_{\mathbb{C}} Z\right)+J_{\mathbb{C}} \nwarrow_{A_{\mathbb{C}}}\left(J_{\mathbb{C}} X, Y, J_{\mathbb{C}} Z\right) \\
= & 4 i \nwarrow_{A_{\mathbb{C}}}(X, Y, Z)-3 J_{\mathbb{C}} \nwarrow_{A_{\mathbb{C}}}(X, Y, Z)=i \nwarrow_{A_{\mathbb{C}}}(X, Y, Z) \\
= & J_{\mathbb{C}} \nwarrow_{A_{\mathbb{C}}}(X, Y, Z),
\end{aligned}
$$

which implies that $J_{\mathbb{C}}$ satisfies (4.77) for all $X, Y, Z \in \mathfrak{q}$. Using a similar computation, we can check that $J_{\mathbb{C}}$ satisfies (4.78) for any $X, Y, Z \in \mathfrak{q}$. Similarly, we can show that $J_{\mathbb{C}}$ satisfies (4.77) and (4.78), for all $\mathcal{X}, \mathcal{Y}, \mathcal{Z} \in A_{\mathbb{C}}$. Let $J \in g l(A)$ given by

$$
\left.J \triangleq J_{\mathbb{C}}\right|_{A}
$$

$J$ is well-defined. Since $J_{\mathbb{C}}$ satisfies (4.77), (4.78), $J_{\mathbb{C}}^{2}=-i d_{\mathbb{C}}$ and $\alpha_{\mathbb{C}} J_{\mathbb{C}}=J_{\mathbb{C}} \alpha_{\mathbb{C}}$ on $A_{\mathbb{C}}$ then $J$ is a complex structure on $A$.

Lemma 4.13. Let $J$ be an almost complex structure on a real 3-Hom-L-dendriform algebra $(A, \nwarrow, \nearrow, \alpha)$ commuting with $\alpha$. If J satisfies

$$
\begin{aligned}
& J \nwarrow(x, y, z)=\nwarrow(J x, y, z)=\nwarrow(x, y, J z) \\
& J \nearrow(x, y, z)=\nearrow(J x, y, z)=\nearrow(x, J y, z)=\nearrow(x, y, J z), \forall x, y, z \in A .
\end{aligned}
$$

Then $\mathrm{J}$ is a complex structure on A called a strict complex structure.

Proof. Using identity (4.83) and $J^{2}=-i d_{A}$, we obtain

$$
\begin{aligned}
& -\nearrow(J x, J y, J z)+\nearrow(J x, y, z)+\nearrow(x, J y, z)+\nearrow(x, y, J z) \\
& +J(J x, J y, z)+J \nearrow(x, J y, J z)+J \nearrow(J x, y, J z) \\
= & -\nearrow(J x, J y, J z)+J \nearrow(x, y, z)+\nearrow(x, J y, z)+\nearrow(x, y, J z) \\
& +\nearrow\left(J^{2} x, J y, z\right)+\nearrow(J x, J y, J z)+\nearrow\left(J^{2} x, y, J z\right) \\
= & J \nearrow(x, y, z) .
\end{aligned}
$$

Similarly, we can get (4.82). Thus, we obtain that $J$ is a complex structure on $A$. 
Let $J$ be a complex structure on $A$. Define two new products $\nwarrow_{J_{1}} \nearrow_{J}: \otimes^{3} A \rightarrow A$ by

$$
\begin{aligned}
& \nwarrow_{J}(x, y, z) \triangleq \frac{1}{4}(\nwarrow(x, y, z)-\nwarrow(x, J y, J z)-\nwarrow(J x, y, J z)-\nwarrow(J x, J y, z)), \\
& \nearrow_{J}(x, y, z) \triangleq \frac{1}{4}(\nearrow(x, y, z)-\nearrow(x, J y, J z)-\nearrow(J x, y, J z)-\nearrow(J x, J y, z)), \quad \forall x, y, z \in A .
\end{aligned}
$$

Proposition 4.14. Let $J$ be a complex structure on a real 3-Hom-L-dendriform algebra $(A, \nwarrow, \nearrow, \alpha)$. Then the tuple $\left(A, \nwarrow_{J}, \nearrow_{J}, \alpha\right)$ is a real 3-Hom-L-dendriform algebra. Moreover, $J$ is a strict complex structure on $\left(A, \nwarrow_{J}, \nearrow_{J}, \alpha\right)$ and the corresponding complex 3-Hom-L-dendriform algebra $\left(A, \nwarrow_{J}, \nearrow_{J}, \alpha\right)$ is isomorphic to the complex 3-Hom-L-dendriform algebra q define in Theorem 4.12

Proof. We will first prove that $\left(A, \nwarrow_{J}, \nearrow_{J}, \alpha\right)$ is a real 3-Hom-L-dendriform algebra. To minimize the computations, we use a new approach to do this. Since $J$ is complex structure on $A$. We can define two map $\varphi: A \rightarrow \mathfrak{q}$ as following:

$$
\varphi(x)=\frac{1}{2}(x-i J x)
$$

It is straightforward to deduce that $\varphi$ is complex linear isomorphism between complex vector spaces.

Since $J \alpha=\alpha J$, then $\varphi \alpha=\alpha \varphi$. By (4.77), for all $x, y, z \in A$, we have

$$
\begin{aligned}
\nwarrow(\varphi(x), \varphi(y), \varphi(z))= & \frac{1}{8} \nwarrow(x-i J x, y-i J y, z-i J z) \\
= & \frac{1}{8}(\nwarrow(x, y, z)-\nwarrow(x, J y, J z)-\nwarrow(J x, y, J z)-\nwarrow(J x, J y, z)) \\
& -\frac{1}{8} i(\nwarrow(x, y, J z)+\nwarrow(x, J y, z)+\nwarrow(J x, y, z)-\nwarrow(J x, J y, J z)) \\
= & \frac{1}{8}(\nwarrow(x, y, z)-\nwarrow(x, J y, J z)-\nwarrow(J x, y, J z)-\nwarrow(J x, J y, z)) \\
& -\frac{1}{8} i J(\nwarrow(x, y, z)-\nwarrow(x, J y, J z)-\nwarrow(J x, y, J z)-\nwarrow(J x, J y, z)) \\
= & \varphi \nwarrow_{J}(x, y, z) .
\end{aligned}
$$

Thus, we have $\nwarrow_{J}(x, y, z)=\varphi^{-1}\left(\nwarrow(\varphi(x), \varphi(y), \varphi(z))\right.$. Similarly, we get $\nearrow_{J}(x, y, z)=\varphi^{-1} \nearrow$ $(\varphi(x), \varphi(y), \varphi(z))$. Since $J$ is a complex structure, $\mathfrak{q}$ is a 3-Hom-L-dendriform subalgebra. Hence $\left(A, \nwarrow_{J}, \nearrow_{J}, \alpha\right)$ is a real 3-Hom-L-dendriform algebra.

By (4.83), for all $x, y, z \in A$, we have

$$
\begin{aligned}
J \nwarrow_{J}(x, y, z) & =\frac{1}{4} J\left(\nwarrow(x, y, z)-\nwarrow_{J}(x, J y, J z)-\nwarrow_{J}(J x, y, J z)-\nwarrow_{J}(J x, J y, z)\right) \\
& =\frac{1}{4}\left(-\nwarrow_{J}(J x, J y, J z)+\nwarrow_{J}(J x, y, z)+\nwarrow_{J}(x, J y, z)+\nwarrow_{J}(x, y, J z)\right) \\
& =\frac{1}{4}\left(-\nwarrow_{J}(J x, J y, J z)+\nwarrow_{J}(J x, y, z)-\nwarrow_{J}\left(J^{2} x, J y, z\right)-\nwarrow_{J}\left(J^{2} x, y, J z\right)\right) \\
& =\nwarrow_{J}(J x, y, z),
\end{aligned}
$$

Similarly, we can check that

$$
J \nwarrow(x, y, z)=\nwarrow(x, y, J z) \text { and } J \nearrow(x, y, z)=\nearrow(J x, y, z)=\nearrow(x, J y, z)=\nearrow(x, y, J z), \forall x, y, z \in A .
$$


which implies that $J$ is a strict complex structure on $\left(A, \nwarrow_{J}, \nearrow_{J}, \alpha\right)$. Identity (4.86) means that $\left(A, \nwarrow_{J}\right.$ $\left., \nearrow_{J}, \alpha\right)$ is isomorphic to the complex 3-Hom-L-dendriform algebra $\mathfrak{q}$.

Proposition 4.15. Let J be a complex structure on a real 3-Hom-L-dendriform algebra $(A, \nwarrow, \nearrow, \alpha)$. Then $J$ is a strict complex structure on $(A, \nwarrow, \nearrow, \alpha)$ if and only if $\nwarrow_{J}=\nwarrow$ and $\nearrow J_{J}=\nearrow$.

Proof. If $J$ is a strict complex structure on $(A, \nwarrow, \nearrow, \alpha)$, by $J \nwarrow(x, y, z)=\nwarrow(J x, y, z)=\nwarrow(x, y, J z)$, we have

$$
\nwarrow_{J}(x, y, z)=\frac{1}{4}(\nwarrow(x, y, z)-\nwarrow(x, J y, J z)-\nwarrow(J x, y, J z)-\nwarrow(J x, J y, z))=\nwarrow(x, y, z) .
$$

Similarly, we can check that $\nearrow_{J}(x, y, z)=\nearrow(x, y, z)$.

Conversely, if $\nwarrow_{J}=\nwarrow$ and $\nearrow_{J}=\nearrow$, we have

$$
-3 \nwarrow(x, y, z)=\nwarrow(x, J y, J z)+\nwarrow(J x, y, J z)+\nwarrow(J x, J y, z) .
$$

Then by condition (4.77), we obtain

$$
\begin{aligned}
4 J \nwarrow_{J}(x, y, z) & =-\nwarrow(J x, J y, J z)+\nwarrow(J x, y, z)+\nwarrow(x, J y, z)+\nwarrow(x, y, J z) \\
& =3 \nwarrow(J x, y, z)+\nwarrow(J x, y, z) \\
& =4 \nwarrow(J x, y, z),
\end{aligned}
$$

which implies that $J \nwarrow(x, y, z)=\nwarrow(J x, y, z)$. Similarly, using (4.78), we obtain $J \nearrow(x, y, z)=\nearrow$ $(J x, y, z)$. The proof is finished.

Lemma 4.16. Let $J$ be an almost complex structure on a real 3-Hom-L-dendriform algebra $(A, \nwarrow, \nearrow, \alpha)$ commuting with $\alpha$. If J satisfies

$$
\begin{aligned}
& \nwarrow(x, y, z)=\nwarrow(x, J y, J z)+\nwarrow(J x, y, J z)+\nwarrow(J x, J y, z), \\
& \nearrow(x, y, z)=\nearrow(x, J y, J z)+\nearrow(J x, y, J z)+\nearrow(J x, J y, z), \forall x, y, z \in A .
\end{aligned}
$$

Then $J$ is a complex structure on A called an abelian complex structure.

Proof. By (4.87) and $J^{2}=-i d_{A}$, we have

$$
\begin{aligned}
& -\nwarrow(J x, J y, J z)+\nwarrow(J x, y, z)+\nwarrow(x, J y, z)+\nwarrow(x, y, J z) \\
& +J \nwarrow(J x, J y, z)+J \nwarrow(x, J y, J z)+J \nwarrow(J x, y, J z) \\
= & -\nwarrow\left(J x, J^{2} y, J^{2} z\right)-\nwarrow\left(J^{2} x, J y, J^{2} z\right)-\nwarrow\left(J^{2} x, J^{2} y, J z\right) \\
& +\nwarrow(J x, y, z)+\nwarrow(x, J y, z)+\nwarrow(x, y, J z)+J \nwarrow(x, y, z) \\
= & J \nwarrow(x, y, z) .
\end{aligned}
$$

Using (4.88), we can prove the condition (4.78). Thus, we obtain that $J$ is a complex structure on A.

Lemma 4.17. Let $J$ be an almost complex structure on a real 3-Hom-L-dendriform algebra $(A, \nwarrow, \nearrow, \alpha)$ commuting with $\alpha$. If J satisfies

$$
\begin{aligned}
& \nwarrow(x, y, z)=-J(\nwarrow(x, y, J z)+\nwarrow(J x, y, z)+\nwarrow(x, J y, z)), \\
& \nearrow(x, y, z)=-J(\nearrow(x, y, J z)+\nearrow(J x, y, z)+\nearrow(x, J y, z)), \forall x, y, z \in A .
\end{aligned}
$$

Then $J$ is a complex structure on A called an strong complex structure. 
Proof. By (4.89) and $J^{2}=-i d_{A}$, we have

$$
\begin{aligned}
& -\nwarrow(J x, J y, J z)+\nwarrow(J x, y, z)+\nwarrow(x, J y, z)+\nwarrow(x, y, J z) \\
& +J \nwarrow(J x, J y, z)+J \nwarrow(x, J y, J z)+J \nwarrow(J x, y, J z) \\
= & J \nwarrow\left(J^{2} x, J y, J z\right)+J \nwarrow\left(J x, J^{2} y, J z\right)+J \nwarrow\left(J x, J y, J^{2} z\right)+J \nwarrow(x, y, z) \\
& +J \nwarrow(J x, J y, z)+J \nwarrow(x, J y, J z)+J \nwarrow(J x, y, J z) \\
= & J \nwarrow(x, y, z) .
\end{aligned}
$$

Using (4.90), we can prove the condition (4.78). Thus, $J$ is a complex structure on $A$.

Lemma 4.18. Let $J$ be an almost complex structure on a real 3-Hom-L-dendriform algebra $(A, \nwarrow, \nearrow, \alpha)$ commuting wilth $\alpha$. If J satisfies

$$
J \nwarrow(x, y, z)=-\nwarrow(J x, J y, J z), J \nearrow(x, y, z)=-\nearrow(J x, J y, J z), \forall x, y, z \in A .
$$

Then $E$ is a complex structure on A called a perfect complex structure.

Proof. By (4.91) and $J^{2}=-i d_{A}$, we have

$$
\begin{aligned}
& -\nwarrow(J x, J y, J z)+\nwarrow(J x, y, z)+\nwarrow(x, J y, z)+\nwarrow(x, y, J z) \\
& +J \nwarrow(J x, J y, z)+J \nwarrow(x, J y, J z)+J \nwarrow(J x, y, J z) \\
= & J \nwarrow(x, y, z)+\nwarrow(J x, y, z)+\nwarrow(x, J y, z)+\nwarrow(x, y, J z) \\
& -\nwarrow\left(J^{2} x, J^{2} y, J z\right)-\nwarrow\left(J x, J^{2} y, J^{2} z\right)-\nwarrow\left(J^{2} x, J y, J^{2} z\right) \\
= & J \nwarrow(x, y, z)
\end{aligned}
$$

and

$$
\begin{aligned}
& -\nearrow(J x, J y, J z)+\nearrow(J x, y, z)+\nearrow(x, J y, z)+\nearrow(x, y, J z) \\
& +J \nearrow(J x, J y, z)+J \nearrow(x, J y, J z)+J \nearrow(J x, y, J z) \\
= & J \nearrow(x, y, z)+\nearrow(J x, y, z)+\nearrow(x, J y, z)+\nearrow(x, y, J z) \\
& -\nearrow\left(J^{2} x, J^{2} y, J z\right)-\nearrow\left(J x, J^{2} y, J^{2} z\right)-\nearrow\left(J^{2} x, J y, J^{2} z\right) \\
= & J \nearrow(x, y, z) .
\end{aligned}
$$

Thus, $J$ is a complex structure on $A$.

The following result illustrates the relation between a complex structure and a product structure on a complex 3-Hom-L-dendriform algebra.

Proposition 4.19. Let $(A, \nwarrow, \nearrow, \alpha)$ be a complex 3-Hom-L-dendriform algebra. Then $E$ is a product structure on $A$ if and only if $J=i E$ is a complex structure on $A$.

Proof. Let $E$ be a product structure on $A$. We have $J^{2}=i^{2} E^{2}=-i d_{A}$. Thus, $J$ is an almost complex structure on $A$. Since $E$ satisfies the condition (4.67), we have

$$
\begin{aligned}
J \nwarrow(x, y, z)= & i E \nwarrow(x, y, z) \\
= & -\nwarrow(i E x, i E y, i E z)+\nwarrow(i E x, y, z)+\nwarrow(x, i E y, z)+\nwarrow(x, y, i E z) \\
& +i E \nwarrow(i E x, i E y, z)+i E \nwarrow(x, i E y, i E z)+i E \nwarrow(i E x, y, i E z) \\
= & -\nwarrow(J x, J y, J z)+\nwarrow(J x, y, z)+\nwarrow(x, J y, z)+\nwarrow(x, y, J z)
\end{aligned}
$$




$$
+J \nwarrow(J x, J y, z)+J \nwarrow(x, J y, J z)+J \nwarrow(J x, y, J z)
$$

Similarly, using (4.68) we can check that

$$
\begin{aligned}
J \nearrow(x, y, z)= & -\nearrow(J x, J y, J z)+\nearrow(J x, y, z)+\nearrow(x, J y, z)+\nearrow(x, y, J z) \\
& +J \nearrow(J x, J y, z)+J \nearrow(x, J y, J z)+J \nearrow(J x, y, J z) .
\end{aligned}
$$

Thus, $J$ is a complex structure on the complex 3-Hom-L-dendriform algebra $A$.

The converse part can be proved similarly and we omit details.

\section{References}

[1] M. Aguiar, Pre-Poisson algebras, Lett. Math. Phys. 54 (2000), no. 4, 263-277.

[2] F. Ammar, S. Mabrouk, A. Makhlouf, Representations and cohomology of n-ary multiplicative Hom-Nambu-Lie algebras. J. of Geom. and Phy. 61 (2011) 1898-1913.

[3] F. Ammar, Z. Ejbehi and Makhlouf, Cohomology and Deformations of Hom-algebras, J. Lie 395 Theory, Vol. 21 (4): 813-836, 2011.

[4] A. Andrada, M. L. Barberis, I. Dotti, Classification of abelian complex structures on 6dimensional Lie algebras, J. of the London Math. Soc. 83.1 (2011): 232-255.

[5] A. Andrada, M. L. Barberis, I. Dotti, G. Ovando, Product structures on four dimensional solvable Lie algebras, Homology, Homotopy and Applications 7(1) (2005): 9-37.

[6] A. Andrada, Complex product structures on 6-dimensional nilpotent Lie algebras, Forum Math. Vol. 20. No. 2. De Gruyter, 2008.

[7] J. Arnlind, A. Makhlouf, S. Silvestrov, Ternary Hom-Nambu-Lie algebras induced by HomLie algebras. Journal of Mathematical Physics, 51(4), 043515(2010).

[8] H. Ataguema,A. Makhlouf, S. Silvestrov, Generalization of n-ary Nambu algebras and beyond. Journal of Mathematical Physics, 50(8), 083501(2009).

[9] J. A. de Azcrraga and J. M. Izquierdo, On a class of n-Leibniz deformations of the simple Filippov algebras, J. Math. Phys. 52, 023521 (2011)

[10] S. Benayadi and A. Makhlouf, Hom-Lie algebras with symmetric invariant nondegenerate bilinear form, J. Geom. Phys. 76 (2014), 38-60.

[11] T. Chtioui, S. Mabrouk, 3-L-dendriform algebras and generalized derivations. arXiv preprint arXiv:2004.05998(2020).

[12] Y. Chen, Z. Wang and L. Zhang, Quasitriangular Hom-Lie bialgebras, J. Lie Theory 22 (2012), 1075-1089.

[13] Y. Chen, H. Zheng and L. Zhang, Double Hom-Associative Algebra and Double Hom-Lie Bialgebra,Adv. Appl. Clifford Algebra 30 (2020), Paper No. 8.

[14] Y. Chen and L. Zhang, Hom-O-operators and Hom-Yang-Baxter equations, Adv. Math. Phys. (2015), Art.ID 823756, 11 pp. 1 
[15] Y. Chen and L. Zhang, Hom-Yang-Baxter equations and Frobenius monoidal Hom-algebras, Adv. Math.Phys. (2018), Art. ID 2912578, 10 pp.

[16] A. Das, Cohomology and deformations of hom-dendriform algebras and coalgebras. arXiv preprint arXiv:2005.14249(2020).

[17] A. Das, Cohomology and deformations of dendriform algebras, and Dend ${ }_{\infty}$ algebras, arXiv preprint,arXiv:1903.11802

[18] A. Das, Cohomology and deformations of dendriform coalgebras, arXiv preprint, arXiv:1907.08255

[19] J. Figueroa-O'Farrill, Deformations of 3-algebras. J. Math. Phys. 50 (2009), no. 11,113514.

[20] V. T. Filippov, n-Lie algebras, Sib. Mat. Zh. 26 (1985), 126-140.

[21] J. Hartwig, D. Larsson, and S. Silvestrov, Deformations of Lie algebras using $\sigma$-derivations, J.Algebra, Vol. 295: 314-361, 2006.

[22] A. Makhlouf, Hom-dendriform algebras and Rota-Baxter Hom-algebras, Operads and universal algebra,147-171, Nankai Ser. Pure Appl. Math. Theoret. Phys., 9, World Sci. Publ., Hackensack, NJ, 2012.

[23] T. Ma and H. Zheng, Some results on Rota-Baxter monoidal Hom-algebras, Results Math. 72 (2017), no. 1-2, 145-170.

[24] J.L. Loday, Dialgebra, Dialgebras and related operads, 7-66, Lecture Notes in Math., 1763, Springer, Berlin, 2001

[25] J.L. Loday and M. Ronco, Trialgebras and families of polytopes, in Homotopy Theory: relations with Algebraic Geometry , Group Cohomology, and Algebraic K-Theory, in Contemp. Math. vol 346, Amer. Math. Soc., Providence, RI, 2004, 369-673.

[26] L. Guo, B. Zhang and S. Zheng, Universal enveloping algebras and Poincaré-Birkhoff-Witt theorem for involutive Hom-Lie algebras, J. Lie Theory 26 (2018), 739-759.

[27] S. Guo, X. Zhang, S. Wang, The constructions of 3-Hom-Lie bialgebras. arXiv preprint arXiv:1902.03917(2019).

[28] D. Larsson and S. D. Silvestrov, Quasi-hom-Lie algebras, central extensions and 2-cocyclelike identities, J. Algebra 288 (2005), 321-344.

[29] L. Liu, A. Makhlouf, C. Menini and F. Panaite, (s, t)-Rota-Baxter operators, infinitesimal Hombialgebras and the associative (Bi)Hom-Yang-Baxter equation, Canad. Math. Bull. 62 (2019), 355-372.

[30] T. Ma and H. Zheng, (m, n)-Hom-Lie algebras, Publ. Math. Debrecen 92 (2018), 59-78.

[31] A. Makhlouf and S. Silvestrov, Hom-algebra structures, J. Gen. Lie Theory Appl., Vol. 2(2): 51-64, 4102008.

[32] A. Makhlouf and S. Silvestrov, Notes on Formal deformations of Hom-Associative and Hom-Lie algebras, Forum Mathematicum, Vol. 22(4): 715-759, 2010. 
[33] Y. Sheng, Representations of hom-Lie algebras, Algebr. Represent. Theory 15 (2012), 10811098.

[34] Y. Sheng, R. Tang, Symplectic, product and complex structures on 3-Lie algebras, J. Algebra. 508 (2018): 256-300.

[35] Y. Sheng and C. Bai, A new approach to hom-Lie bialgebras, J. Algebra 399 (2014), 232-250.

[36] Y. Sheng and D. Chen, Hom-Lie 2-algebras, J. Algebra 376 (2013), 174-195.

[37] L. Song and R. Tang, Derivation Hom-Lie 2-algebras and non-abelian extensions of regular Hom-Lie algebras, J. Algebra Appl. 17 (2018), 1850081.

[38] Q. Wang, Y. Sheng, C. Bai,J. Liu, Nijenhuis operators on pre-Lie algebras. Communications in Contemporary Mathematics, 21(07), 1850050 (2019).

[39] S. Wang and S. Wang, Hom-Lie algebras in Yetter-Drinfeld categories, Comm. Algebra 42 (2014), 4526- 4547.

[40] D. Yau, Hom-algebras and homology, J. Lie Theory 19 (2009), 409-421.

[41] D. Yau, Hom-algebras and homology, J.Lie Theory, Vol. 19: 409-421, 2009.

[42] D. Yau, The Hom-Yang-Baxter equation and Hom-Lie algebras, J. Math. Phys. 52 (2011), 053502.

[43] J. Zhao, L. Chen and L. Ma, Representations and T*-extensions of hom-Jordan-Lie algebras, Comm. Algebra 44 (2016), 2786-2812.

[44] S. Zheng and L. Guo, Free involutive Hom-semigroups and Hom-associative algebras, Front. Math. China 11 (2016), 497-508.

[45] T. Zhang, Deformations and Extensions of 3-Lie Algebras, arXiv:1401.4656. 